\title{
COSBO: THE MAMBO 1.2 MILLIMETER IMAGING SURVEY OF THE COSMOS FIELD ${ }^{1}$
}

\author{
F. Bertoldi, ${ }^{2}$ C. Carilli, ${ }^{3}$ M. Aravena, ${ }^{2,4}$ E. Schinnerer,${ }^{5}$ H. Voss,${ }^{4}$ V. Smolcic, ${ }^{5}$ K. Jahnke, ${ }^{5}$ N. Scoville, ${ }^{6}$ \\ A. Blain, ${ }^{6}$ K. M. Menten, ${ }^{4}$ D. Lutz, ${ }^{7}$ M. Brusa, ${ }^{7}$ Y. Taniguchi, ${ }^{8}$ P. CapaK, ${ }^{6}$ B. Mobasher,${ }^{6}$ \\ S. Lilly, ${ }^{9}$ D. Thompson, ${ }^{6}, 10$ H. Aussel, ${ }^{11,12}$ E. Kreysa, ${ }^{4}$ G. Hasinger, ${ }^{7}$ \\ J. Aguirre, ${ }^{13}$ J. Schlaerth, ${ }^{13}$ and A. Koekemoer ${ }^{14}$ \\ Received 2006 September 22; accepted 2007 April 25
}

\begin{abstract}
The inner $20 \times 20 \operatorname{arcmin}^{2}$ of the COSMOS field was imaged at $250 \mathrm{GHz}(1.2 \mathrm{~mm})$ to an rms noise level of $\sim 1 \mathrm{mJy}$ per $11^{\prime \prime}$ beam using the Max-Planck Millimeter Bolometer Array (MAMBO-2) at the IRAM $30 \mathrm{~m}$ telescope. We detect 15 sources at significance between 4 and $7 \sigma, 11$ of which are also detected at $1.4 \mathrm{GHz}$ with the VLA with a flux density $>24 \mu \mathrm{Jy}(3 \sigma)$. We identify 12 more lower significance $\mathrm{mm}$ sources based on their association with faint radio sources. We present the multifrequency identifications of the MAMBO sources, including VLA radio flux densities, optical and near-infrared identifications, as well as the XMM-Newton X-ray detection for two of the mm sources. We compare radio and optical photometric redshifts and briefly describe the host galaxy morphologies. The colors of the identified optical counterparts suggest most of them to be high-redshift $(z \sim 2-3)$ star-forming galaxies. At least three sources appear lensed by a foreground galaxy. We highlight some MAMBO sources that do not show obvious radio counterparts. These sources could be dusty starburst galaxies at redshifts $>3.5$. The $250 \mathrm{GHz}$ source areal density in the COSMOS field is comparable to that seen in other deep mm fields.
\end{abstract}

Subject headings: submillimeter — infrared: galaxies — surveys

Online material: color figure

\section{INTRODUCTION}

Submillimeter and millimeter wide field imaging surveys with SCUBA and MAMBO have revealed a population of active starforming galaxies at high redshifts (see Blain et al. 2002 for an early review and for more recent surveys: Scott et al. 2002; Greve et al. 2004; Coppin et al. 2006; Pope et al. 2006). These objects dominate the far-IR background, and current models suggest that this population may represent the formation of spheroidal galaxies at $z$ between 2 and 5. With the discovery of this dust-obscured population of star-forming galaxies, (sub-) millimeter surveys lead to a significant revision to the star formation history of the universe derived from optical surveys. The (sub-) $\mathrm{mm}$ population is estimated to constitute a significant fraction of cosmic star formation from the big bang to the present (Dunlop 2001).

Determining the redshift distribution for (sub-) mm galaxies has been of paramount importance since their first discovery. The

\footnotetext{
${ }^{1}$ Based on observations with the $30 \mathrm{~m}$ telescope of the Institute for Radioastronomy at Millimeter Wavelengths (IRAM), which is funded by the German Max-Planck-Society, the French CNRS, and the Spanish National Geographical Institute. Also based on observations with the Very Large Arrray of the National Radio Astronomy Observatory, which is a facility of the National Science Foundation, operated under cooperative agreement by Associated University Inc. Based on observations with the NASA/ESA Hubble Space Telescope, obtained at the Space Telescope Science Institute, which is operated by the Association of Universities for Research in Astronomy, Inc. (AURA), under NASA contract NAS 5-26555; also based on data collected at the Subaru Telescope, which is operated by the National Astronomical Observatory of Japan; XMM-Newton, an ESA science mission with instruments and contributions directly funded by ESA Member States and NASA; the European Southern Observatory, Chile; Kitt Peak National Observatory, Cerro Tololo Inter-American Observatory, and the National Optical Astronomy Observatory, which are operated by the Association of Universities for Research in Astronomy, Inc. (AURA), under cooperative agreement with the National Science Foundation; and the Canada-France-Hawaii Telescope operated by the National Research Council of Canada, Centre National de la Recherche Scientifique de France and the University of Hawaii.

2 Argelander Institute for Astronomy, University of Bonn, Auf dem Hügel 71, 53121 Bonn, Germany.

${ }_{3}^{3}$ National Radio Astronomy Observatory, P.O. Box, Socorro, NM 87801.
}

principal issue has been the low resolution of the (sub-) $\mathrm{mm}$ images, and the resulting confusion when trying to identify optical counterparts. One standard method for determining at least a gross redshift estimate for (sub-) mm galaxies has been to use the radioFIR correlation (e.g., Carilli \& Yun 1999). Chapman et al. (2003) have refined this approach further by using the radio sources to localize the submillimeter galaxy (confusion in the radio is a minor concern) and thereby to identify them with optical and/or near-IR (NIR) counterparts that can be targeted with optical spectroscopy.

Deep optical/NIR imaging and spectroscopy and high-resolution CO follow-up observations (Genzel et al. 2003; Neri et al. 2003; Greve et al. 2005; Tacconi et al. 2006) of SCUBA and MAMBO sources have shown them to be massive starburst galaxies, often with coexisting active galactic nuclei (AGNs) of minor luminosity (Alexander et al. 2005). Many of them display disturbed structure indicative of galaxy interaction or merger (e.g., Chapman et al. 2004; Genzel et al. 2003). The high observed average space density of the massive (sub-) mm background starbursts

\footnotetext{
4 Max-Planck-Institute for Radioastronomie, Auf dem Hügel 69, 53121 Bonn, Germany.

5 Max-Planck-Institute for Astronomy, Königstuhl 17, 69117 Heidelberg, Germany.

${ }^{6}$ California Institute of Technology, Pasadena, CA 91125.

7 Max-Planck-Institute for extraterrestrial Physics, Giessenbachstraße, D-85748 Garching, Germany.

${ }^{8}$ Physics Department, Graduate School of Science and Engineering, Ehime University, 2-5 Bunkyo-cho, Matsuyama 790-8577, Japan.

9 Department of Physics, Swiss Federal Institute of Technology, ETH Hönggerberg, CH-8093 Zurich, Switzerland.

10 Large Binocular Telescope Observatory, University of Arizona, 933 North Cherry Avenue, Tucson, AZ 85721.

${ }_{11}$ Institute for Astronomy, University of Hawaii, 2680 Woodlawn Drive, Honolulu, HI 96822

12 Service d'Astrophysique, CEA/Saclay, 91191 Gif-sur-Yvette, France.

${ }^{13}$ Center for Astrophysics and Space Astronomy and Department of Astronomy and Planetary Sciences, University of Colorado, 593 UCB, Boulder, CO 80303-0593.

${ }^{14}$ Space Telescope Science Institute, 3700 San Martin Drive, Baltimore, MD 21218
} 
places tight constraints on our understanding of galaxy formation, forcing models to account that a large fraction $(\sim 10 \%)$ of all baryons that reside in massive halos assembled into galaxies very early on (Greve et al. 2005).

Most of the detailed studies of (sub-) mm background sources have focused on those identified also at radio wavelengths (e.g., Ivison et al. 2005, 2007), thus providing accurate positions and showing optical counterparts. However, it was found that many of the (sub-) mm galaxies are optically faint and can be seen only in very deep NIR images (Dannerbauer et al. 2004). Their colors are such that they must lie either at very high redshifts, or their colors are distinctly different from those of local starburst galaxies such as Arp 220. These findings are based on only a handful of objects that were securely identified with high-resolution $\mathrm{mm}$ interferometry and deep ISAAC or Keck NIR imaging (Bertoldi et al. 2000; Dannerbauer et al. 2004). Much of the work so far has been biased toward $z<3$ radio-identified (sub-) $\mathrm{mm}$ sources, although the highest redshift massive starbursts place the tightest constraints on galaxy formation models. A major aim of recent (sub-) $\mathrm{mm}$ surveys has been to increase the number of sources and try to identify objects at higher redshifts using deep radio or infrared imaging.

\subsection{COSMOS}

Beyond a redshift of $\sim 0.5$ there are as yet few constraints on the relation between the large-scale cosmic mass distribution that provides the environment for galaxy assembly, and the properties of galaxies, such as their morphology, size, and age. Galaxy evolution and the morphological mix are thought to be strongly dependent on galaxy environment, i.e., large-scale structure (LSS), but this is well established only for the local universe through, e.g., the $2 \mathrm{dF}$ and Sloan surveys. Substantial LSS occurs on scales up to $\sim 100 \mathrm{Mpc}$ (comoving), including voids, filaments, groups, and clusters (Peacock et al. 2001; Schuecker et al. 2001). Adequately mapping galaxy evolution over the full range of environments and redshifts therefore requires data covering wide areas coupled with accurate spectroscopic redshifts for the line-of-sight discrimination in the LSS.

The COSMOS HST imaging program has been designed to cover a wide area $1.4^{\circ} \times 1.4^{\circ}$ to address these issues (Scoville et al. 2007). An equatorial field has been chosen to allow for easy access with all ground-based facilities. Observations were done or are underway at essentially all wavelengths. The COSMOS field will be the definitive field for constraining models of galaxy formation and LSS for the foreseeable future.

In this paper, we present preliminary results of our ongoing COSMOS imaging survey of far-IR dust continuum emission from high- $z$ galaxies using the MAMBO bolometer array at the IRAM $30 \mathrm{~m}$ telescope. This MAMBO program (dubbed COSBO) entails a sensitive (sub-mJy) survey at $1.2 \mathrm{~mm}$ of the inner $\sim 20 \times$ $20 \mathrm{arcmin}^{2}$ of the COSMOS field. The MAMBO program is complemented by a wider area but shallower $1.1 \mathrm{~mm}$ Bolocam survey (J. E. Aguirre et al. 2007, in preparation).

\section{OBSERVATIONS AND RESULTS}

\subsection{MAMBO Observations}

To image the COSMOS field at wavelength $1.2 \mathrm{~mm}$, we used the 117 channel Max-Planck Bolometer array (MAMBO-2; Kreysa et al. 1998) at the IRAM $30 \mathrm{~m}$ telescope on Pico Veleta, Spain. MAMBO-2 has a half-power spectral bandwidth from 210 to $290 \mathrm{GHz}$, with an effective bandwidth center for steep thermal spectra of $\sim 250 \mathrm{GHz}(1.20 \mathrm{~mm})$. The effective beam FWHM is $10.7^{\prime \prime}$, and the undersampled field of view is $4^{\prime}$. Our observations

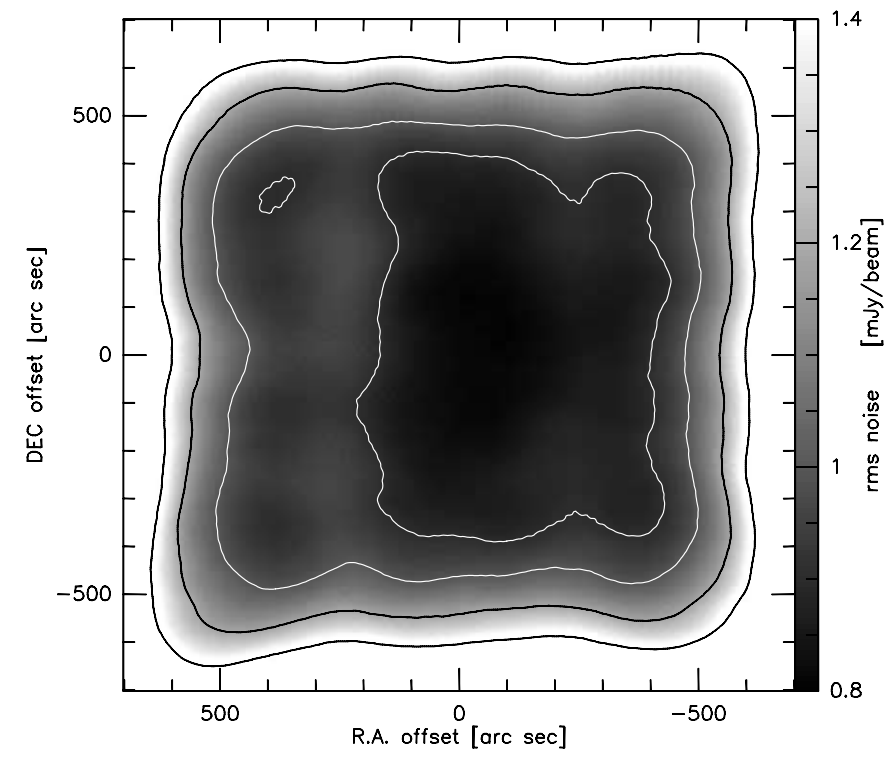

FIG. 1.- Root mean square noise distribution in the COSBO map. Contours indicate $0.9,1.0,1.2$, and $1.5 \mathrm{mJy}$ per $11^{\prime \prime}$ beam.

were done within pooled observing runs spread over the winters of 2003-2004 and 2004-2005. They continued in the winters of 2005-2006 and 2006-2007; however, these data are not included here. Atmospheric conditions were generally good during the observations, with typical zenith opacities between 0.1 and 0.35 and low sky noise. The total on sky integration time was $78 \mathrm{hr}$. We used the standard on-the-fly mapping technique, where one map is comprised of 41 azimuthal subscans of $60 \mathrm{~s}$ each, while chopping the secondary mirror by $42^{\prime \prime}-46^{\prime \prime}$ in azimuth at $2 \mathrm{~Hz}$. We employed a grid of evenly spaced offsets to cover an expanding field to nearly uniform depth (Fig. 1). The telescope pointing was checked about once per hour using the bright point source QSO J0909+014 and was found to be stable to within $3^{\prime \prime}$. The absolute flux calibration is based on observations of several standard calibration sources, including planets, and the resulting flux calibration uncertainty is estimated to be about $10 \%(\mathrm{rms})$. The data were analyzed using the MOPSI and MOPSIC software packages written by R. Zylka at IRAM.

Correlated sky noise was subtracted by forming weighted averages of the signals of surrounding channels. The double-beam maps were combined using a shift-and-add procedure, producing for each map a positive source image bracketed by two negative images of half the intensity located one chop throw away. Because beam chopping is in the azimuth direction, each single map covering a source produces a shift-and-add triple image pattern that is inclined in the equatorial co-added map, depending on hour angle. The "point spread function" is thereby different at every point in the final map, and although it could be reconstructed from the scan information, gains, and opacities, we do not attempt to deconvolve sources with the local PSF. Because the source density is low at the current survey depth, the effect of confusion due to the PSF negative sidelobes is not significant. The noise level increases toward the edge of our co-added map due to decreasing exposure time. Figure 1 plots the noise distribution (per 10.7" beam), showing areas of $\sim 450$ and $\sim 250 \operatorname{arcmin}^{2}$ to $<2$ and $<1 \mathrm{mJy}$, respectively.

\subsection{Source Extraction}

Sources were extracted from the mosaicked map by fitting elliptical Gaussians, using MOPSIC's gf it routine (cf. Greve et al. 


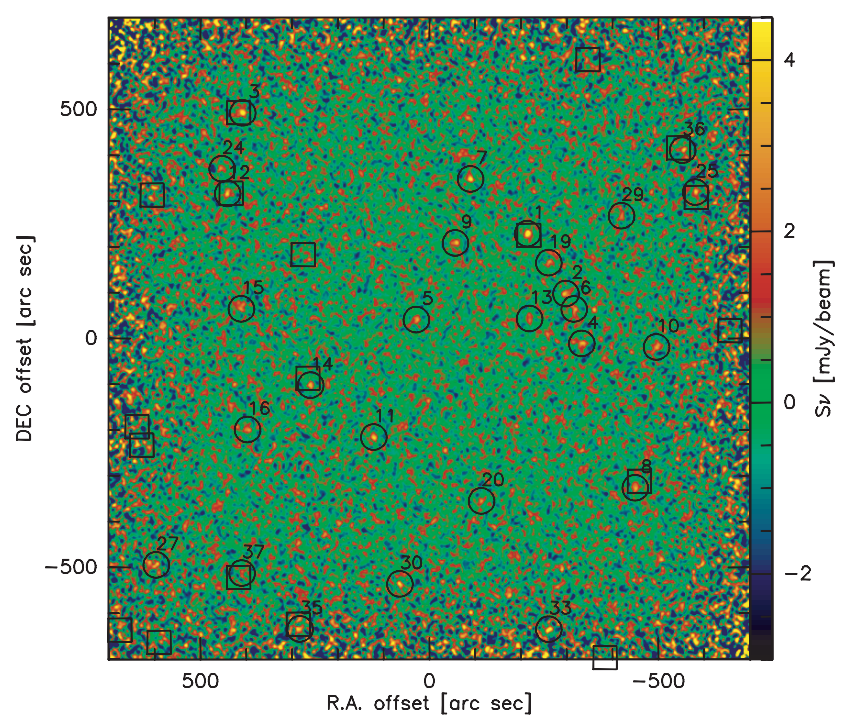

FIG. 2.- Inner part of the MAMBO $1.2 \mathrm{~mm}$ map smoothed to $12^{\prime \prime}$ resolution from the original 10.7". Detected sources are circled with ID numbers. Squares mark the Bolocam $\mathrm{S} / \mathrm{N}>3.5$ sources from Aguirre et al. (2007). Offsets are in arcsec from R.A. $10^{\mathrm{h}} 00^{\mathrm{m}} 30^{\mathrm{s}}$, decl. $+02^{\circ} 12^{\prime} 00^{\prime \prime}(\mathrm{J} 2000.0)$. Note that the noise level rises toward the map edges.

$2005)$. The fit accuracy often depends on the initial guess of the fitting parameters and on the region around the source included in the fit. We used an IDL routine that automatically locates all peaks in the signal-to-noise map above a given threshold and locates all contiguous pixels around a given peak with values above a fixed, lower threshold. From these pixels the area of the map used for the Gaussian fit is set, as well as the initial Gaussian parameter guess. In some cases a manual intervention was necessary to converge at an acceptable result. The integrated flux density of the fitted Gaussians is then renormalized to a circular Gaussian having the full width at half-maximum (FWHM) of the nominal $10.7^{\prime \prime}$ beam.

In the COSMOS MAMBO map we thereby identified 15 sources with a signal-to-noise ratio $(\mathrm{S} / \mathrm{N})>4$ (Figs. 2 and 6 , Table 1 ) in the integrated flux density. In some cases, a MAMBO source appears fragmented or elongated in the $11^{\prime \prime}$ resolution map, possibly due to a combination of noise and pointing errors, in some cases maybe due to multiplicity. The selection of a diffuse MAMBO peak as a single source might therefore be somewhat subjective. For some of the more diffuse sources the peak flux density remains below $\mathrm{S} / \mathrm{N}=4$ in the $11^{\prime \prime}$ or $12^{\prime \prime}$ smoothed maps, while the integrated flux has $\mathrm{S} / \mathrm{N}>4$.

From Gaussian source fitting we see marginal evidence for source extension for some of the fainter sources. However, a convolution of the map to lower resolution usually does not confirm this and may thus hint at a combination of low signal-to-noise and confusion with background structure.

The source fluxes must be corrected for the effect of "flux boosting" (Scott et al. 2002; Coppin et al. 2006), also known as (or confused with) Malmquist or Eddington bias. It arises from instrumental, atmospheric, and confusion noise that scatters the measured source flux and leads to an average flux overestimate in a sensitivity-limited survey, and in a bias in the number counts. To partially correct for this effect, we follow the approach of Greve et al. (2004): we produced maps from our data with randomized (i.e., wrong) array parameters. Thereby the residual, correlated noise over the array and the average source background is preserved, while the individual source intensity information is lost. Before reducing the randomized maps we added point sources with a range of flux densities. On the final map we used the same source extraction algorithm as on the original map to measure the source fluxes, $S_{\text {out }}$, and compared these with the input flux densities, $S_{\text {in }}$ (Fig. 3). For our COSMOS data the boosting factor is best fitted by $S_{\text {out }} / S_{\text {in }}=1+1.7 e^{-0.75 S_{\text {in }}}$, which we used to correct the extracted source flux densities for flux boosting (Table 1). Since the boosting fraction depends on the source $\mathrm{S} / \mathrm{N}$, we limited our test to the map area with noise level 0.9-1.2 mJy, which includes most of our extracted sources.

\subsection{Multiwavelength Association}

\subsubsection{Radio Counterparts}

Past millimeter and submillimeter surveys have shown that a reliable method to locate the mm source to within a few arcsec is to identify a corresponding faint $1.4 \mathrm{GHz}$ radio source near the mm peak (e.g., Dannerbauer et al. 2004; Ivison et al. 2005). From the strong correlation between the far-IR and radio luminosities that is found in star-forming galaxies (Condon 1992), we expect a radio flux density of order 10 to $100 \mu \mathrm{Jy}$ for mm sources of several mJy at redshifts 1 to 3 . The much lower abundance of faint radio sources (about $0.8 \mathrm{arcmin}^{-2}$ with $S_{1.4 \mathrm{GHz}}>24 \mu \mathrm{Jy}$ ) compared with optical sources (about $400 \mathrm{arcmin}^{-2}$ in the COSMOS point source catalog) makes a chance association of a mm source with a radio source unlikely, although at the 3-4 $\sigma$ level in the radio data, fiducial sources might be wrongly identified. The radio identification of (sub-) mm sources has even been used to estimate the positional uncertainty of the (sub-) mm source positions, estimated as the mean separation between the VLA radio and MAMBO sources. Previous MAMBO surveys here yield a scatter of at most $\sim 5^{\prime \prime}$ (e.g., Dannerbauer et al. 2004; Ivison et al. 2005).

To identify possible radio counterparts to the MAMBO sources we used deep (7-8 $\mu \mathrm{Jy}$ rms, $1.35^{\prime \prime}$ round beam) VLA $1.4 \mathrm{GHz}$ observations that include data we obtained in addition to the VLACOSMOS large project data $(10 \mu \mathrm{Jy})$ presented by Schinnerer et al. (2004, 2007).

We limited the search for likely radio counterparts of the $\mathrm{mm}$ sources to an $8^{\prime \prime}$ radius around the MAMBO position, accounting for the MAMBO positional uncertainty due to beam size $\left(\sim 11^{\prime \prime}\right)$ and pointing errors $\left(\sim 3^{\prime \prime}\right)$. The significance of an association is estimated in the common way, by computing the corrected Poisson probability that a radio source is found by chance in a background of randomly distributed radio sources (Downes et al. 1986; see also Ivison et al. 2002, 2005). For each of the most likely radio counterparts to a MAMBO source we computed the corrected Poisson probability $P_{c}=1-\exp \left\{P_{S}\left[1+\log \left(P_{S} / P_{3 \sigma}\right)\right]\right\}$, where $P_{S}=1-\exp \left[-\pi n(S) d^{2}\right]$ is the "raw probability" to find a source brighter $S$ within a distance $d$ from the mm source, $n_{S}$ being the local density of radio sources brighter than the candidate. Correspondingly, $P_{3 \sigma}$ shall be the raw probability to find a source brighter than our detection limit within the search radius of $8^{\prime \prime}$. Table 1 lists $P_{c}$ for each radio candidate and Figure 4 shows the distribution of their $P_{c}$ compared with that of a random position sampling in the radio map. Our comparison with a random sample shows that most of the radio identifications are likely to be real.

For the $15 \mathrm{~S} / \mathrm{N}>4 \mathrm{MAMBO}$ sources we found in the COSMOS field, 4 do not show a radio source above our $3 \sigma$ threshold of $25 \mu \mathrm{Jy}$ (sources 4, 9, 14, 15; Fig. 6). Of the 11 detected, at least 6 show extended emission at the resolution $\left(1.35^{\prime \prime}\right)$ of our VLA data.

In addition to the unbiased $>4 \sigma \mathrm{MAMBO}$ source selection, we have identified 3-4 $\sigma$ MAMBO peaks that coincide well (within $5^{\prime \prime}$ ) with radio peaks and added this radio-selected sample 
TABLE 1

Mambo Sources with Their Possible Radio Counterparts

\begin{tabular}{|c|c|c|c|c|c|c|c|c|c|c|c|}
\hline \multirow[b]{3}{*}{ ID } & \multirow{3}{*}{$\begin{array}{l}\text { NAME } \\
(\mathrm{MM} \mathrm{J})\end{array}$} & \multirow{2}{*}{\multicolumn{2}{|c|}{ MAMBO PosITION }} & \multirow{3}{*}{$\begin{array}{c}S_{250 \mathrm{GHz}}{ }^{\mathrm{a}} \\
(\mathrm{mJy})\end{array}$} & \multicolumn{3}{|c|}{ VLA Position } & \multirow[b]{3}{*}{$P_{c}^{\mathrm{b}}$} & \multirow{3}{*}{$\begin{array}{c}S_{1.4 \mathrm{GHz}}{ }^{\mathrm{c}} \\
(\mu \mathrm{Jy})\end{array}$} & \multirow{2}{*}{\multicolumn{2}{|c|}{ FLAG $^{\mathrm{d}}$}} \\
\hline & & & & & & & Offset to MAMBO & & & & \\
\hline & & R.A. (J2000.0) & Decl. (J2000.0) & & R.A. (J2000.0) & Decl. (J2000.0) & $(\operatorname{arcsec})$ & & & Confidence & Bolocam Name \\
\hline 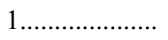 & $100016+021549$ & 100015.7 & 021549 & $(6.3-0.10) \pm 0.9$ & & & & & & & 4 \\
\hline $1 \mathrm{E} \ldots \ldots \ldots \ldots \ldots \ldots$ & & & & & 100015.59 & 021549.6 & 1.8 & 0.0100 & $25 \pm 7.5$ & 0 & \\
\hline $1 \mathrm{~W} \ldots \ldots \ldots \ldots \ldots \ldots$ & & & & & 100015.43 & $02 \quad 1549.6$ & 4.1 & 0.0307 & $27 \pm 7.5$ & 0 & \\
\hline $2 \ldots \ldots \ldots \ldots \ldots \ldots$ & $100010+021338$ & 100010.1 & 021338 & $(5.9-0.13) \pm 0.9$ & & & & & & & \\
\hline $2 \mathrm{~W} \ldots \ldots \ldots \ldots \ldots \ldots$ & & 100009.7 & 021333 & $(2.2-0.83) \pm 0.9$ & 100009.63 & 021334.5 & 1.8 & 0.0173 & $34 \pm 10$ & - & \\
\hline $2 \mathrm{E} \ldots \ldots \ldots \ldots \ldots$ & & 100010.4 & 021337 & $(4.2-0.37) \pm 0.9$ & 100010.13 & 021335.0 & 4.5 & 0.0471 & $60 \pm 10$ & 0 & \\
\hline 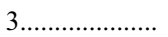 & $100057+022013$ & 100057.2 & 022013 & $(7.5-0.05) \pm 1.1$ & 100057.27 & 022012.6 & 1.1 & 0.0029 & $50 \pm 8$ & + & 33 \\
\hline 4.......................... & $100007+021149$ & 100007.8 & 021149 & $(5.7-0.15) \pm 0.9$ & None & & & & $<24$ & - & \\
\hline $5 \ldots \ldots \ldots \ldots \ldots \ldots$ & $100031+021241$ & 100031.9 & 021241 & $(5.3-0.19) \pm 0.9$ & 100031.82 & 021243.1 & 2.4 & 0.0036 & $105 \pm 7,222 \pm 34$ & + & \\
\hline $6 \ldots \ldots \ldots \ldots \ldots \ldots \ldots$ & $100008+021304$ & 100008.9 & 021304 & $(5.2-0.20) \pm 0.9$ & 100008.80 & 021304.4 & 1.5 & 0.0075 & $70 \pm 8$ & + & \\
\hline 7...................... & $100024+021748$ & 100024.0 & 021748 & $(5.2-0.20) \pm 0.9$ & 100023.97 & 021750.2 & 2.2 & 0.0049 & $51 \pm 8,117 \pm 44$ & + & \\
\hline 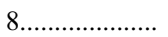 & $100000+020634$ & 100000.0 & 020634 & $(5.6-0.15) \pm 1.0$ & 095959.94 & 020633.3 & 1.1 & 0.0031 & $77 \pm 7.5,97 \pm 25$ & + & 12 \\
\hline 9 & $100026+021529$ & 100026.2 & 021529 & $(4.9-0.24) \pm 0.9$ & None & & & & $<24$ & - & \\
\hline $10 \ldots \ldots \ldots \ldots \ldots \ldots$ & $095956+021139$ & 095956.9 & 021139 & $(6.0-0.12) \pm 1.1$ & & & & & & & \\
\hline 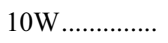 & & & & & 095956.87 & $02 \quad 1137.2$ & 1.9 & 0.0059 & $26 \pm 7.5$ & 0 & \\
\hline $10 \mathrm{E} \ldots \ldots \ldots \ldots . . . .$. & & & & & 095957.07 & 021136.9 & 3.3 & 0.0123 & $50 \pm 7.5$ & 0 & \\
\hline $11 \ldots \ldots \ldots \ldots \ldots$ & $100038+020825$ & 100038.1 & 020825 & $(4.8-0.25) \pm 0.9$ & & & & & & & \\
\hline $11 \mathrm{E} \ldots \ldots \ldots \ldots \ldots . . . .$. & & & & & 100038.02 & 020822.6 & 2.7 & 0.0034 & $175 \pm 8,237 \pm 27$ & 0 & \\
\hline $11 \mathrm{~W} \ldots \ldots \ldots \ldots \ldots$ & & & & & 100037.89 & 020821.9 & 3.9 & 0.0279 & $38 \pm 7$ & 0 & \\
\hline $12 \ldots \ldots \ldots \ldots \ldots \ldots$ & $100059+021716$ & 100059.4 & 021716 & $(5.0-0.22) \pm 1.0$ & & & & & & & \\
\hline $12 \mathrm{~N} \ldots \ldots \ldots \ldots \ldots \ldots$ & & & & & 100059.24 & $02 \quad 17 \quad 19.2$ & 3.9 & 0.0118 & $73 \pm 8,121 \pm 29$ & 0 & 24 \\
\hline $12 \mathrm{C} \ldots \ldots \ldots \ldots \ldots \ldots$ & & & & & 100059.35 & 021715.5 & 0.9 & 0.0044 & $20 \pm 8$ & 0 & \\
\hline $12 \mathrm{~S} \ldots \ldots \ldots \ldots \ldots$ & & & & & 100059.31 & 021710.4 & 5.9 & 0.0321 & $44 \pm 10$ & 0 & \\
\hline $13 \ldots \ldots \ldots \ldots \ldots \ldots$ & $100015+021244$ & 100015.4 & 021244 & $(4.4-0.32) \pm 0.9$ & & & & & & & \\
\hline $13 \mathrm{~N} \ldots \ldots \ldots \ldots \ldots$ & & 100015.5 & 021253 & $(2.3-0.83) \pm 0.9$ & & & & & $<24$ & - & \\
\hline $13 \mathrm{~S} \ldots \ldots \ldots \ldots \ldots$ & & 100015.3 & 021240 & $(2.2-0.83) \pm 0.9$ & 100015.29 & 021240.6 & 0.7 & 0.0002 & $202 \pm 8,348 \pm 31$ & + & \\
\hline $14 \ldots \ldots \ldots \ldots \ldots \ldots \ldots$ & $100047+021018$ & 100047.3 & 021018 & $(4.4-0.32 \pm 1.0$ & 100047.46 & $\begin{array}{lll}02 & 10 & 14.2\end{array}$ & 4.6 & 0.0235 & $<24$ & - & 29 \\
\hline $15 \ldots \ldots \ldots \ldots \ldots \ldots$ & $100057+021305$ & 100057.4 & 021305 & $(4.4-0.32) \pm 1.0$ & None & & & & $<23$ & - & \\
\hline $16 \ldots \ldots \ldots \ldots \ldots \ldots$ & $100056+020841$ & 100056.5 & 020841 & $(3.8-0.45) \pm 1.0$ & 100056.34 & 020839.1 & 3.1 & 0.0117 & $55 \pm 8$ & + & \\
\hline $19 \ldots \ldots \ldots \ldots \ldots \ldots$ & $100012+021445$ & 100012.6 & 021445 & $(3.5-0.55) \pm 0.9$ & 100012.56 & 021444.1 & 1.1 & 0.0110 & $22 \pm 7$ & + & \\
\hline $20 \ldots \ldots \ldots \ldots \ldots \ldots$ & $100022+020605$ & 100022.4 & 020605 & $(3.5-0.55) \pm 0.9$ & 100022.32 & 020605.1 & 1.2 & 0.0024 & $55 \pm 7$ & + & \\
\hline $24 \ldots \ldots \ldots \ldots \ldots . . .$. & $100100+021811$ & 100100.2 & 021811 & $(3.4-0.58) \pm 1.0$ & 100100.22 & 021813.7 & 2.7 & 0.0167 & $39 \pm 7.5$ & + & \\
\hline $25 \ldots \ldots \ldots \ldots \ldots \ldots$ & $095951+021720$ & 095951.2 & 021720 & $(4.5-0.30) \pm 1.3$ & 095951.07 & 021715.7 & 4.7 & 0.0319 & $37 \pm 8$ & + & 19 \\
\hline $27 \ldots \ldots \ldots \ldots \ldots . . .$. & $100109+020346$ & 100109.8 & 020346 & $(4.2-0.37) \pm 1.4$ & 100109.85 & 020346.4 & 0.9 & 0.0026 & $60 \pm 9$ & + & \\
\hline $29 \ldots \ldots \ldots \ldots \ldots \ldots \ldots$ & $100002+021628$ & 100002.0 & 021628 & $(3.6-0.51) \pm 1.2$ & 100001.80 & 021627.6 & 2.9 & 0.0289 & $48 \pm 8$ & 0 & \\
\hline $30 \ldots \ldots \ldots \ldots \ldots \ldots$ & $100034+020304$ & 100034.3 & 020304 & $(3.5-0.54) \pm 1.2$ & 100034.31 & 020302.3 & 1.7 & 0.0060 & $57 \pm 7.5$ & + & \\
\hline 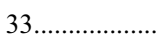 & $100012+020125$ & 100012.6 & 020125 & $(5.2-0.20) \pm 2.0$ & 100012.52 & 020124.2 & 1.4 & 0.0060 & $35 \pm 8$ & + & \\
\hline $35 \ldots \ldots \ldots \ldots \ldots . . .$. & $100048+020125$ & 100049.0 & 020126 & $(4.4-0.32) \pm 1.5$ & 100048.89 & 020125.4 & 1.8 & 0.0081 & $39 \pm 8$ & + & 15 \\
\hline $36 \mathrm{E} \ldots \ldots \ldots \ldots \ldots . . . . . . .$. & $095953+021851$ & 095953.9 & 021851 & $(2.2-0.83) \pm 0.9$ & 095953.87 & 021854.0 & 3.3 & 0.0180 & $48 \pm 8$ & + & 9 \\
\hline $36 \mathrm{~W} \ldots \ldots \ldots \ldots \ldots \ldots$ & & 095953.1 & 021851 & $(3.5-0.54) \pm 0.9$ & None & & & & & - & \\
\hline $37 \ldots \ldots \ldots \ldots \ldots \ldots$ & $100057+020228$ & 100057.3 & 020328 & $(2.8-0.74) \pm 1.1$ & 100057.21 & 020322.4 & 5.8 & 0.0200 & $121 \pm 7.5$ & + & 6 \\
\hline
\end{tabular}

Note.-Units of right ascension are hours, minutes, and seconds, and units of declination are degrees, arcminutes, and arcseconds.

aAMBO flux density is source-integrated flux normalized to $1.7^{\prime \prime}$ beam, followed by flux boosting correction and the rms noise level around the source as estimate for the measurement error.

Raw poisson probability for a chance association of the radio source with the MAMBO source center.

${ }^{\mathrm{c}}$ If one flux density is given, it is the peak value; if two, the second is integrated.

d Flag indicates confidence of radio identification of the MAMBO source: (+) yes; (0) ambiguous; (-) no. Last column gives Bolocam source name (Aguirre et al. 2007). 


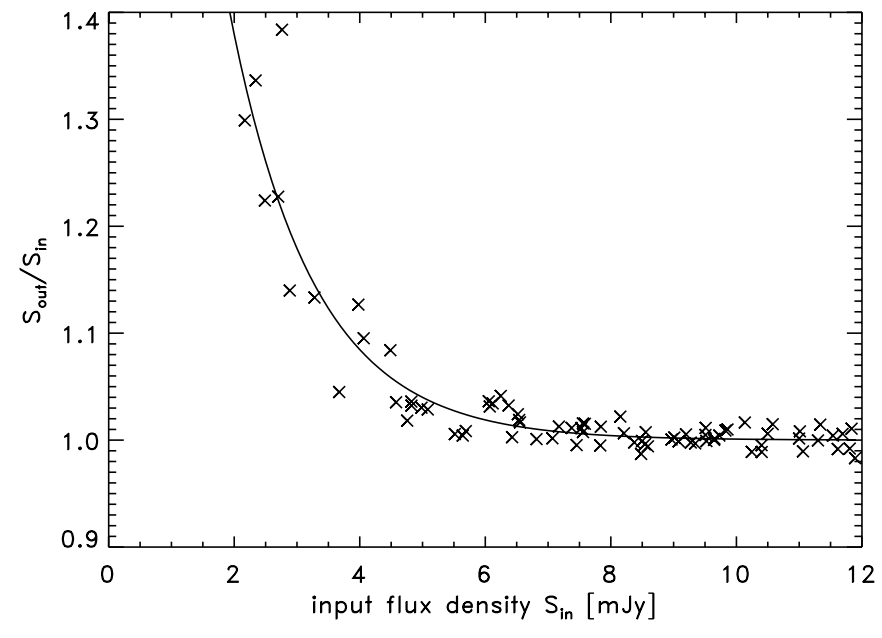

FIG. 3.-Flux boosting of artificial point sources that were added to our randomized MAMBO maps. Shown is the ratio between extracted and input flux as a function of input flux density, and a fit to the data points. At an apparent extracted flux of $(2,3,4,5) \mathrm{mJy}$, the statistical correction for flux boosting is $-(0.76,0.70$, $0.41,0.22) \mathrm{mJy}$.

of 10 sources to our source list (Table 1). We furthermore added two sources $(36,37)$ that have $<4 \sigma$ significance in the MAMBO map, but coincide with Bolocam sources, and lead by the Bolocam position, also with radio sources.

The distribution of offsets between the radio candidate counterparts and the MAMBO sources (Fig. 5) is well in the range of the MAMBO source position uncertainty and is similar to that found by, e.g., Ivison et al. (2005). With about $1 / 30$ spurious radio source within $8^{\prime \prime}$, the expected contamination by random associations is small.

\subsubsection{Optical Counterparts}

For the identification of optical sources to the likely radio counterparts we used imaging data from deep Subaru $i^{+}$-band (Taniguchi et al. 2007), HST ACS $I$-band, and $K$-band (KPNO $4 \mathrm{~m}$ ) observations, as well as searching the COSMOS point source catalog (Capak et al. 2007), which is mostly based on the Subaru data. Our optical data have $5 \sigma$ point source detection limits of

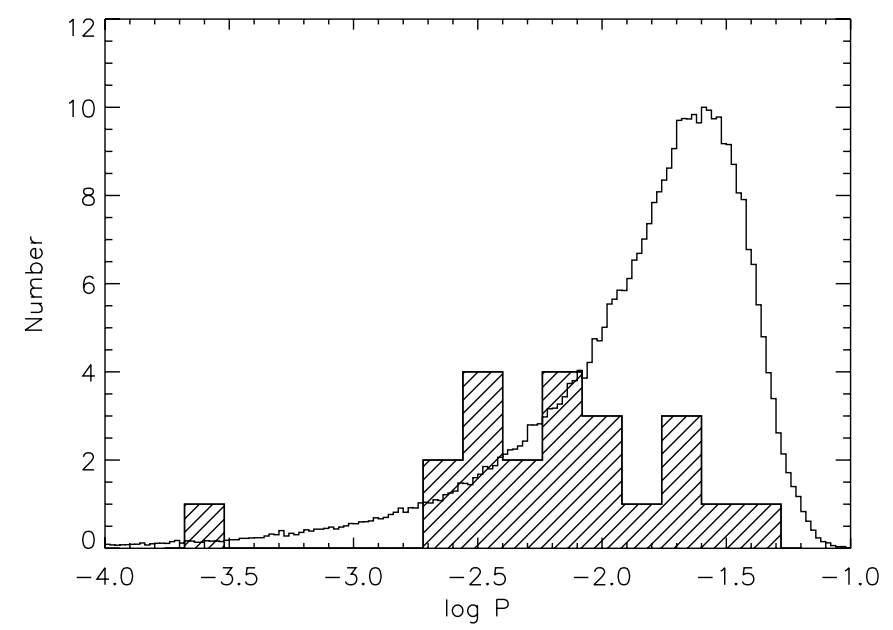

FIG. 4.-Distribution of the corrected Poisson probability of a chance association of the most likely radio counterparts to the MAMBO sources (shaded histogram), compared with the distribution found from a Monte Carlo simulation for which the reference position is randomly chosen on the radio map. The cutoff at $P \sim 0.05$ is due to the limiting search radius of $11^{\prime}$ and the depth of our COSMOS radio map.

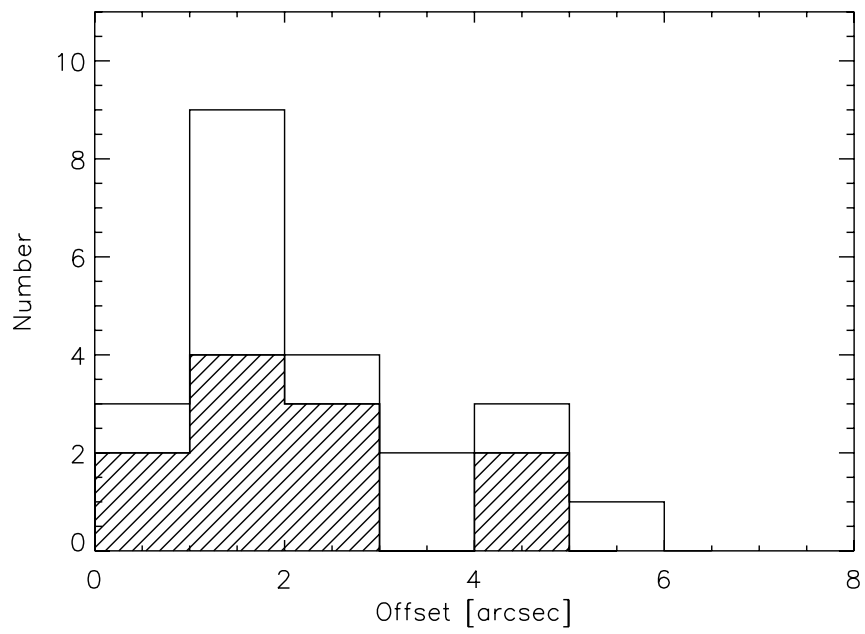

FIG. 5.- Offset between the position of the most likely radio counterpart and the MAMBO source center position. The shaded histogram shows the most likely counterparts among the millimeter-selected sources $1-15$. On top, we show the radio-selected mm sources. With an areal density of $0.8 \mathrm{arcmin}^{-2}$ for radio sources with $S_{1.4 \mathrm{GHz}}>24 \mu \mathrm{Jy}$, we can expect one spurious association among the 24 radio sources identified.

26.9 mag (AB, Subaru $i^{+}$band; Taniguchi et al. 2007), $27 \mathrm{mag}$ (AB, HST ACS $I$ band), $19.7 \mathrm{mag}$ (Vega, $K$ band), $21 \mathrm{mag}$ (AB, KPNO 4 m FLAMINGOS, in aperture twice the seeing FWHM).

Within a MAMBO beam of $\sim 11^{\prime \prime}$ we typically find many sources in the deep optical images ( $\sim 10$ COSMOS PSC sources), none of which may necessarily be the counterpart to the mm emission. In near-IR images the source density is lower, but even there an unambiguous identification is difficult. We searched for optical counterparts of the radio sources that we find most likely to be the MAMBO counterparts (Table 2; Fig. 6).

Because of the high spatial resolution of the VLA, Subaru, and ACS images, we select optical counterparts as object in the Subaru or ACS images that spatially overlap with the radio source image, or as entries in the COSMOS point source catalog within $0.5^{\prime \prime}$ from the radio source center position, assuring a $<10 \%$ chance for a spurious identification.

For the sources without a likely radio counterpart, we do not attempt to identify an optical counterpart. We have scheduled mminterferometric observations to better locate (and confirm) these sources and will try an identification using new ground-based near-IR and Spitzer observations in a subsequent publication.

\subsubsection{X-Ray Counterparts}

We cross-correlated our MAMBO sources with the X-ray source counterparts from the XMM-Newton observations (Hasinger et al. 2007; Brusa et al. 2007). We found two possible X-ray-MAMBO associations: MAMBO source 11 with XID 160 and source 20 with XID 278 (Table 3). The X-ray counterparts for these two sources were identified with ID 366459 and ID 286874 from the photoredshift catalog (Mobasher et al. 2007). Furthermore, we would tentatively associate source 29 with XID 13, which is a very bright $\mathrm{X}$-ray source, but the counterpart to the $\mathrm{X}$-ray source is $\sim 8^{\prime \prime}$ apart from the one we tentatively associate with the MAMBO and VLA source.

\subsubsection{Bolocam Comparison}

We compared our MAMBO map with the source list of a shallower $\left(\sigma \approx 1.9 \mathrm{mJy}\right.$ per $31^{\prime \prime}$ FWHM beam $)$ and wider $\left(31^{\prime} \times 31^{\prime}\right)$ Bolocam $1.1 \mathrm{~mm}$ survey (Aguirre et al. 2007). Eight of the most significant 15 Bolocam sources $(>3.5 \sigma)$ are covered in the 
TABLE 2

Radio Identifications with Possible Optical Counterparts

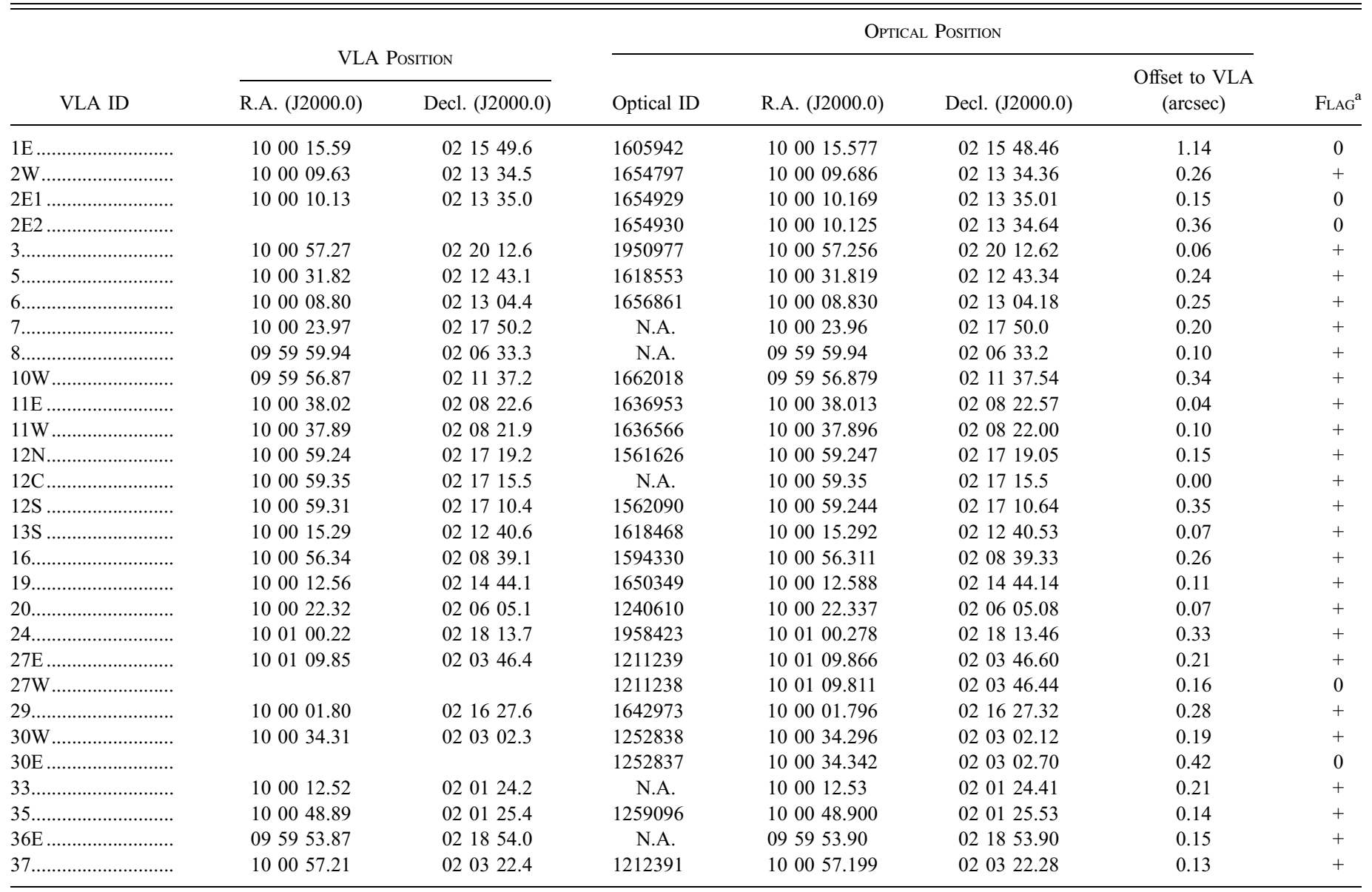

Note.-Units of right ascension are hours, minutes, and seconds, and units of declination are degrees, arcminutes, and arcseconds.

${ }^{a}$ Flag indicates confidence of optical identification of the radio source: $(+)$ clear; $(0)$ ambiguous or questionable.

MAMBO map, and four of these have significant MAMBO counterparts (Table 4) within the positional uncertainty of the respective surveys. The others are mostly in the noisier edge regions and the MAMBO upper flux limits are consistent with the Bolocam flux densities. Ten of the 23 less significant (3-3.5 $\sigma$ ) Bolocam sources are covered by the MAMBO map, and four of those are detected with MAMBO at $>3.5 \sigma$; the others have upper flux limits consistent with the Bolocam fluxes. Of the 35 MAMBO sources identified at $>3.5 \sigma$, only 7 were detected $(>3 \sigma)$ with Bolocam. We have measured the Bolocam map flux density at the position of those 28 MAMBO sources not detected there and find that only 7 of them show flux levels (measured Bolocam map flux plus noise rms) inconsistent with the measured MAMBO flux (measured flux). Overall, from the 35 MAMBO and Bolocam sources in the MAMBO field, 8 were detected in both surveys, 7 have inconsistent fluxes, and the remaining 20 sources have consistent upper flux limits in the respectively other map. The positional offsets of the MAMBO and Bolocam cross-identified sources is between $5^{\prime \prime}$ and $17^{\prime \prime}$, which is consistent with the positional uncertainties expected from the MAMBO (11") and Bolocam (31") beams and the typically low $\mathrm{S} / \mathrm{N}$ of the detections.

\subsection{Photometric Redshifts}

We have calculated radio photometric redshift estimates using the VLA and MAMBO (boosting-corrected) fluxes, assuming the relationship derived for star-forming galaxies by Carilli \& Yun $(1999,2000)$ (Table 5). They are based on the tight radio-FIR correlation found in star-forming galaxies (Condon 1992), with the added stipulation of a standard cm-to-FIR SED characteristic of an active star-forming galaxy. The uncertainty range we quote for the radio redshifts reflects only the measurement uncertainty of the radio-mm spectral index but not that of the Carilli \& Yun model. The latter would typically add an uncertainty of $+1 /-0.5$. The radio-mm redshifts can serve as an orientation that an object is either at low or high redshift, but considering the inherent uncertainties, arising e.g., from the dust properties, it is not very reliable.

Photometric redshifts were also obtained from optical and nearIR photometry for the possible optical/NIR counterparts of those radio sources most likely associated with the MAMBO sources (Table 2). We have not attempted to identify possible optical counterparts to MAMBO sources for which no radio counterpart could be identified.

The COSMOS source and photoredshift catalogs (Mobasher et al. 2007; Capak et al. 2007) list two photometric redshifts, one obtained with the Mobasher et al. (2007) code and another with the Benítez (2000) BPZ code. Both were found from $\chi^{2}$ minimizations using six templates: $\mathrm{E} / \mathrm{S} 0, \mathrm{Sab}, \mathrm{Sc}, \mathrm{Im}$, and two starburst. The main difference between the two is that the Mobasher code includes reddening, while the BPZ code does not. In addition to the published catalog values, we reapplied Mobasher's code with a different setup: we allowed for redshifts up to 6.5 rather than 
01
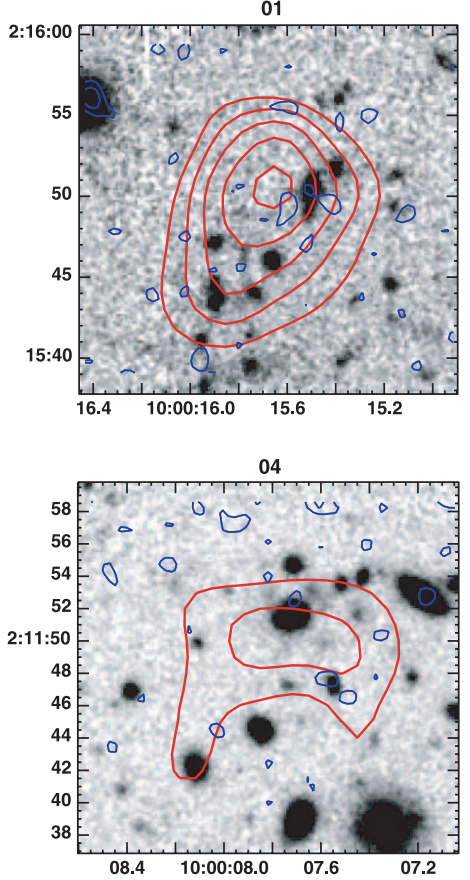

07

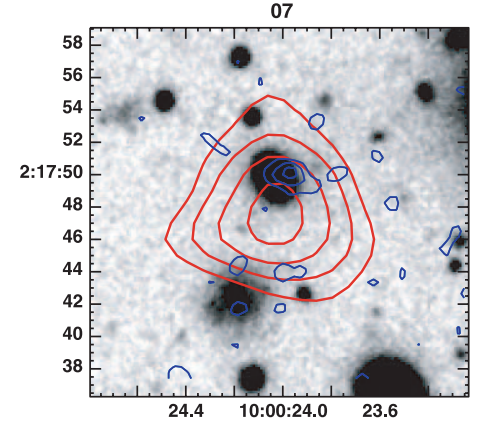

10

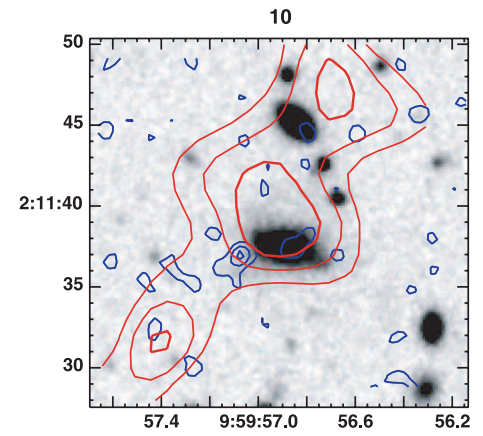

02

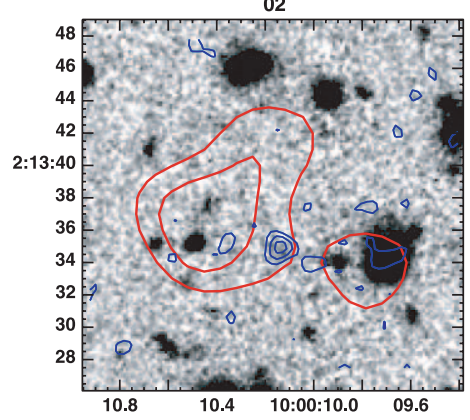

05

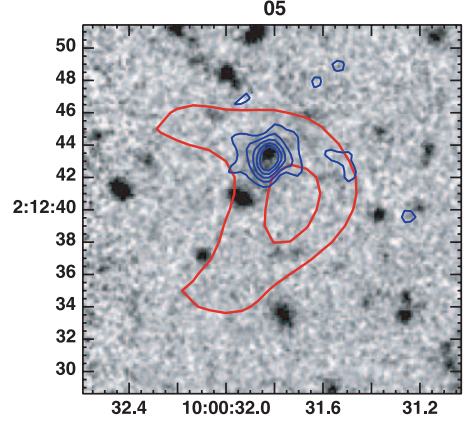

08
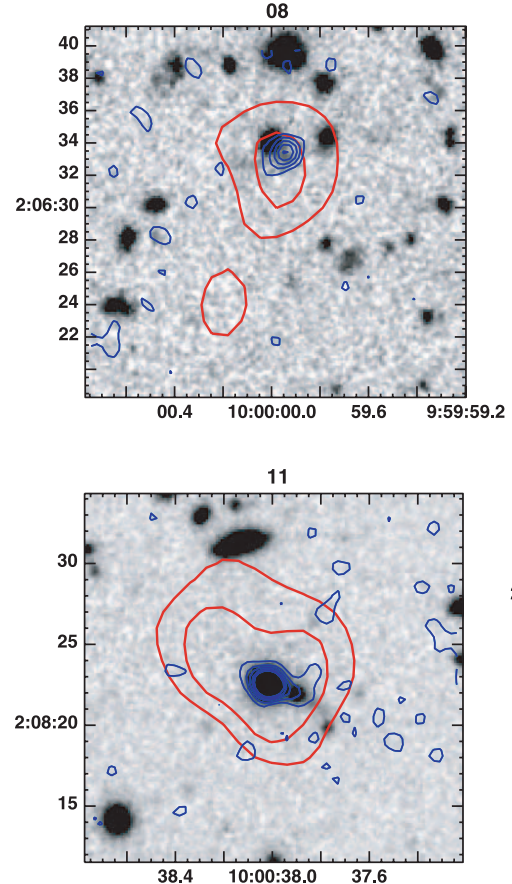

03

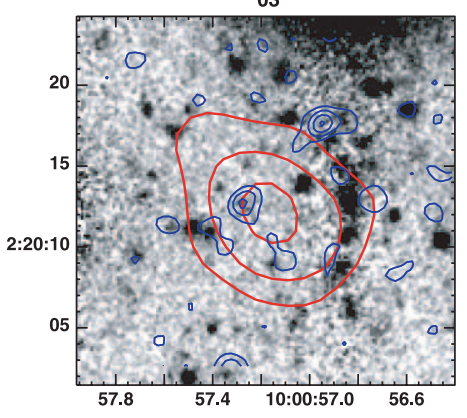

06

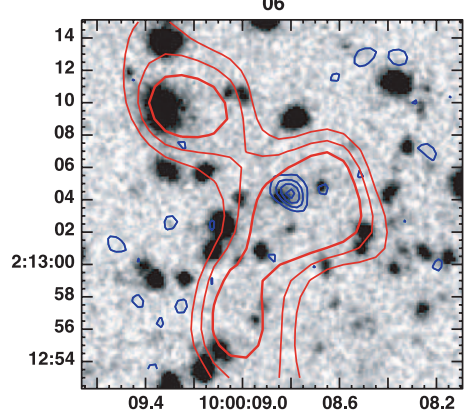

09

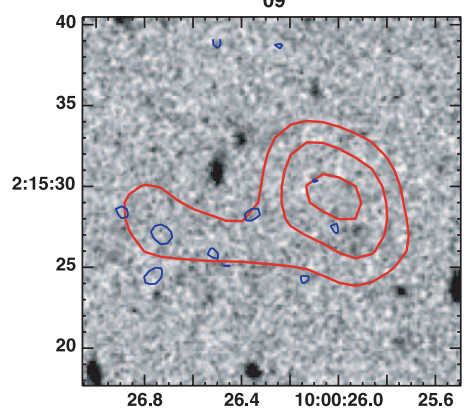

12

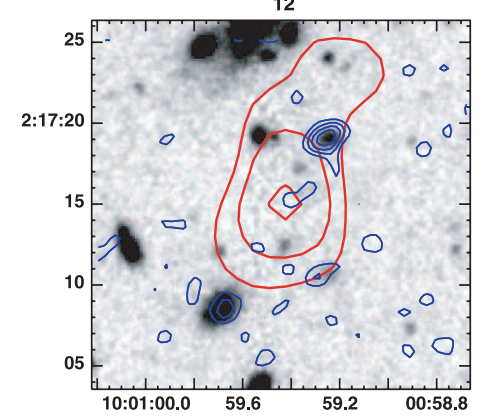

FIG. 6. - Images $\left(25^{\prime \prime} \times 25^{\prime \prime}\right)$ centered on the MAMBO source and labeled by the MAMBO source ID number. The gray-scale $i^{\prime}$-band Subaru images are log-scaled and smoothed individually to optimize the contrast. Thick (red) contours represent MAMBO S/N levels $(2.5,3.5,4.5,5.5$, and $6.5 \sigma$, for some sources also 1.5 and $2 \sigma$ contours as thinner lines), and the thin (blue) contours are VLA $1.4 \mathrm{GHz}$ intensity levels $(15,30,45, \ldots \mu \mathrm{Jy})$; FWHM size of the round radio restoring beam is $\left.1.35^{\prime \prime}\right)$.

3 , and for up to 6 mag of visual extinction rather than the $1 \mathrm{mag}$ allowed for in the COSMOS catalog (Fig. 7). Mobasher's code will not calculate photometric redshifts for sources with 3 or fewer photometry points.

In addition to these redshift estimates, we computed the $\chi^{2}(z)$ distributions using the HYPERZ code (Bolzonella et al. 2000). For this we used either the four CWW (Coleman et al. 1980) template spectra (E, Sab, Sbc, Scd) with the complete set of filter bands currently available for COSMOS $\left(u^{\star}, i^{\star}, B_{\mathrm{J}}, V_{\mathrm{J}}, g^{+}, r^{+}\right.$, $i^{+}, z^{+}, K_{s}$, plus the 5 SDSS bands), or alternatively, a set of syn- thetic templates obtained using the GALAXEV03 code (Bruzual $\&$ Charlot 2003) and using the Calzetti et al. (2000) reddening correction. These templates correspond to a short starburst, three exponentially declining star formation rates $(\tau=1,3,15 \mathrm{Gyr})$, and two constant star formation rates $\left(1,10 M_{\odot} \mathrm{yr}^{-1}\right)$.

For many of our possible optical MAMBO counterparts, the minimum in the $\chi^{2}(z)$ distribution is not very pronounced, and several local mimima corresponding to different galaxy templates have only slightly different values of $\chi^{2}$. Since we do not impose priors on the nature of the MAMBO galaxies, for the HYPERZ 

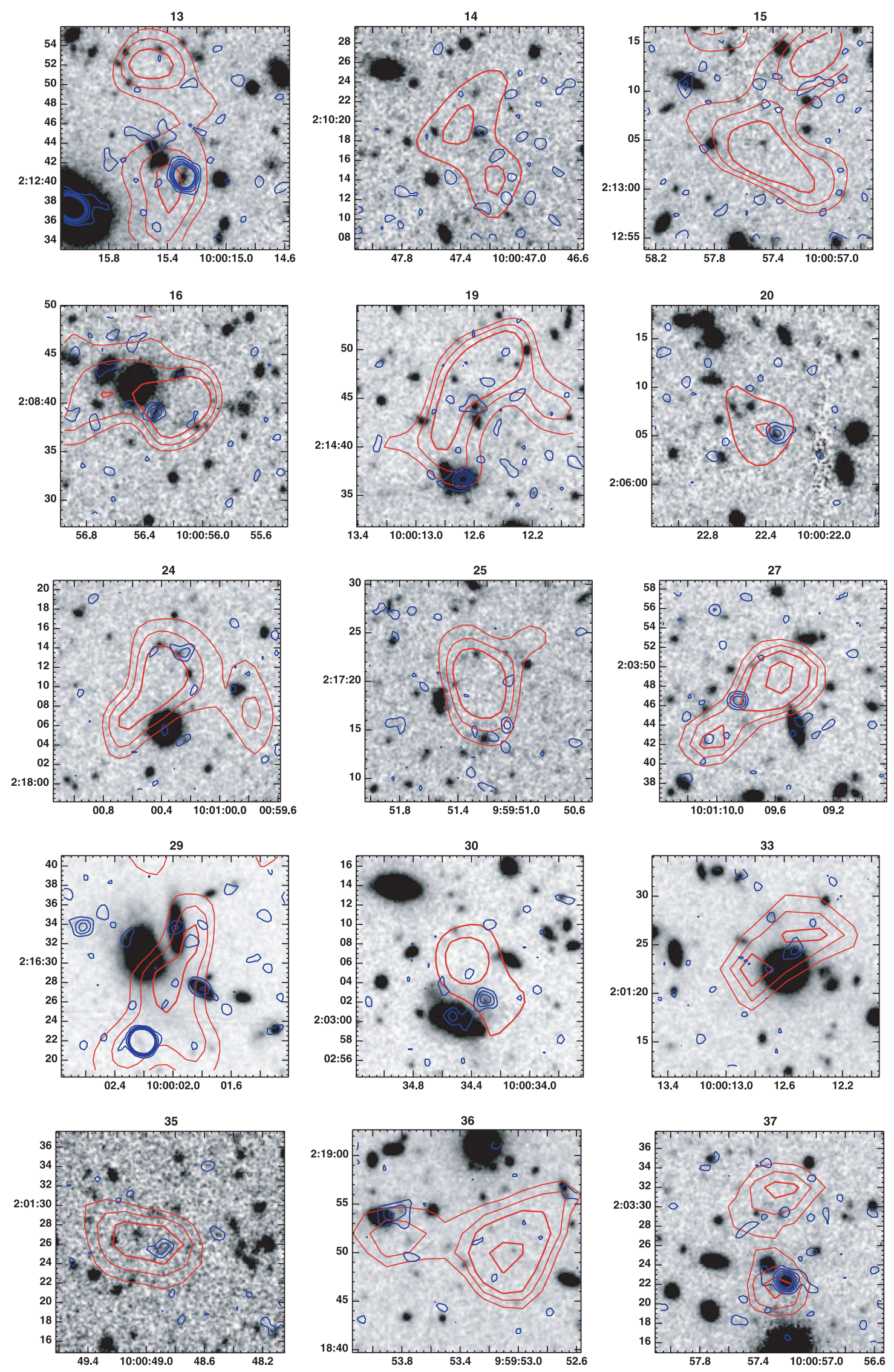

FIG. 6-Continued 
TABLE 3

X-RAY IDENTIFICATIONS

\begin{tabular}{|c|c|c|c|c|c|c|c|c|}
\hline $\mathrm{mm}-\mathrm{ID}$ & X-ID & R.A. (J2000.0) & Decl. (J2000.0) & $\begin{array}{c}\text { Soft } \\
\left(\mathrm{erg} \mathrm{cm}-2 \mathrm{~s}^{-1}\right)\end{array}$ & $\begin{array}{c}\text { Hard } \\
\left(\text { erg } \mathrm{cm}^{-2} \mathrm{~s}^{-1}\right)\end{array}$ & Hardness & $\begin{array}{l}\text { X-Radio } \\
(\operatorname{arcsec})\end{array}$ & $\begin{array}{c}L_{\mathrm{X}}^{\mathrm{a}} \\
\left(\mathrm{erg} \mathrm{s}^{-1}\right)\end{array}$ \\
\hline $11 \mathrm{E}$ & 160 & 100037.9543 & +020822.46 & $2.39 \mathrm{E}-15$ & $1.02 \mathrm{E}-14$ & -0.08 & 1.0 & $2.5 \mathrm{E} 43$ \\
\hline $29 \ldots \ldots \ldots \ldots \ldots \ldots$ & 13 & 100002.2659 & +021631.29 & $2.11 \mathrm{E}-14$ & $2.9 \mathrm{E}-14$ & -0.54 & $\mathrm{~b}$ & \\
\hline
\end{tabular}

Note.- Units of right ascension are hours, minutes, and seconds, and units of declination are degrees, arcminutes, and arcseconds.

${ }^{a}$ The quantity $L_{\mathrm{X}}$ is the full band $[0.5-10 \mathrm{keV}]$ rest frame luminosity in the case of source $11 \mathrm{E}$, and the $[0.5-2 \mathrm{keV}]$ rest frame luminosity for source 20 .

b $\mathrm{X}$-ray source is clearly not related to the radio source.

redshifts we list (Table 5) the range of best and second best redshifts found, which typically correspond to different galaxy types.

To summarize our findings, Table 5 lists the Mobasher, BPZ, and HYPERZ $\chi^{2}$ minimum (maximum likelihood) redshifts, as well as a range of redshifts corresponding to the two lowest $\chi^{2}$

TABLE 4

MAMBO-Bolocam Source Comparison

\begin{tabular}{|c|c|c|c|c|}
\hline \multicolumn{2}{|c|}{ MAMBO } & \multicolumn{2}{|r|}{ BOLOCAM } & \multirow[b]{2}{*}{$\begin{array}{c}\text { Separation } \\
(\operatorname{arcsec})\end{array}$} \\
\hline ID & $\begin{array}{c}S_{1.2 \mathrm{~mm}} \\
(\mathrm{mJy})\end{array}$ & ID & $\begin{array}{c}S_{1.1 \mathrm{~mm}} \\
(\mathrm{mJy})\end{array}$ & \\
\hline 1 ............................ & $(6.3-0.10) \pm 0.9$ & 4 & $(7.8-1.9) \pm 1.9$ & 5 \\
\hline $2 \ldots \ldots \ldots \ldots \ldots \ldots$ & $(5.9-0.13) \pm 0.9$ & & 3.7 & \\
\hline 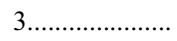 & $(7.5-0.05) \pm 1.1$ & 33 & $(5.9-2.4) \pm 1.9$ & 9 \\
\hline 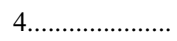 & $(5.7-0.15) \pm 0.9$ & & 4.1 & \\
\hline $5 \ldots \ldots \ldots \ldots \ldots \ldots$ & $(5.3-0.19) \pm 0.9$ & & 3.5 & \\
\hline 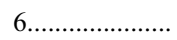 & $(5.2-0.20) \pm 0.9$ & & 3.7 & \\
\hline 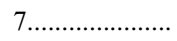 & $(5.2-0.20) \pm 0.9$ & & 1.3 & \\
\hline 8......................... & $(5.6-0.15) \pm 1.0$ & 12 & $(7.1-2.2) \pm 1.9$ & 17 \\
\hline 9........................ & $(4.9-0.24) \pm 0.9$ & & 3.1 & \\
\hline 10...................... & $(6.0-0.12) \pm 1.1$ & & 1.8 & \\
\hline $11 \ldots \ldots \ldots \ldots \ldots \ldots$ & $(4.8-0.25) \pm 0.9$ & & 5.6 & \\
\hline $12 \ldots \ldots \ldots \ldots \ldots \ldots$ & $(5.0-0.22) \pm 1.0$ & 24 & $(6.1-2.4) \pm 1.9$ & 8 \\
\hline $13 \ldots \ldots \ldots \ldots \ldots$ & $(4.4-0.32) \pm 0.9$ & & 4.3 & \\
\hline $14 \ldots \ldots \ldots \ldots \ldots . .$. & $(4.4-0.32) \pm 1.0$ & 29 & $(6.0-2.4) \pm 1.9$ & 16 \\
\hline $15 \ldots \ldots \ldots \ldots \ldots . .$. & $(4.4-0.32) \pm 1.0$ & & 2.2 & \\
\hline $16 \ldots \ldots \ldots \ldots \ldots \ldots$ & $(3.8-0.45) \pm 1.0$ & & 0.6 & \\
\hline $20 \ldots \ldots \ldots \ldots \ldots \ldots \ldots$ & $(3.5-0.55) \pm 0.9$ & & 1.1 & \\
\hline 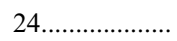 & $(3.4-0.58) \pm 1.0$ & & -0.0 & \\
\hline $25 \ldots \ldots \ldots \ldots \ldots \ldots . .$. & $(4.5-0.30) \pm 1.3$ & 19 & $(6.5-2.3) \pm 1.9$ & 12 \\
\hline $27 \ldots \ldots \ldots \ldots \ldots \ldots . . .$. & $(4.2-0.37) \pm 1.4$ & & 0.8 & \\
\hline $29 \ldots \ldots \ldots \ldots \ldots \ldots . .$. & $(3.6-0.51) \pm 1.2$ & & 3.2 & \\
\hline $30 \ldots \ldots \ldots \ldots \ldots \ldots \ldots$ & $(3.5-0.54) \pm 1.2$ & & -0.9 & \\
\hline $33 \ldots \ldots \ldots \ldots \ldots$ & $(5.2-0.20) \pm 2.0$ & & 4.7 & \\
\hline 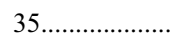 & $(4.4-0.32) \pm 1.3$ & 15 & $(6.7-2.3) \pm 1.9$ & 10 \\
\hline 36......................... & $(4.2-0.37) \pm 1.2$ & 9 & $(7.5-2.1) \pm 1.9$ & 6 \\
\hline $37 \ldots \ldots \ldots \ldots \ldots \ldots$ & $(2.8-0.74) \pm 1.1$ & 6 & $(7.7-2.0) \pm 1.9$ & 12 \\
\hline & $<5.2$ & 2 & $(8.7-1.6) \pm 1.9$ & \\
\hline & $<4.3$ & 10 & $(7.3-2.1) \pm 1.9$ & \\
\hline & $<5.3$ & 11 & $(7.2-2.2) \pm 1.9$ & \\
\hline & $<6.7$ & 16 & $(6.6-2.3) \pm 1.9$ & \\
\hline & $<5.2$ & 18 & $(6.5-2.3) \pm 1.9$ & \\
\hline & $<8.4$ & 25 & $(6.1-2.4) \pm 2.0$ & \\
\hline & $<8.4$ & 28 & $(6.0-2.4) \pm 1.9$ & \\
\hline & $<2.9$ & 30 & $(6.0-2.4) \pm 1.9$ & \\
\hline & $<4.0$ & 31 & $(6.0-2.5) \pm 1.9$ & \\
\hline
\end{tabular}

NoTES.-Flux comparison between all MAMBO and all Bolocam sources in the MAMBO field, listing the integrated source flux minus Eddington bias $\pm \mathrm{rms}$ noise level at the source position. For MAMBO sources with no Bolocam catalog source, the Bolocam map flux found at the MAMBO source position is listed. For Bolocam sources with no MAMBO source, a $3 \sigma$ upper limit is listed. minima of the HYPERZ fits to synthetic or CWW templates. Although there are large uncertainties in both the radio and optical redshifts, they are mostly consistent within these uncertainties.

Three of the securely identified optical counterparts have redshift solutions that are significantly discrepant with another: 24 , $27 \mathrm{~W}$, and 35 . All three galaxies are faint, extended, and irregular on $0.5^{\prime \prime}-1^{\prime \prime}$ scale, which makes the low-redshift $(0.1-0.2)$ solutions unlikely. The low redshifts would also be inconsistent with the radio-mm photometric redshifts.

For one MAMBO optical counterpart (11E) we do have a good optical spectroscopic redshift. Interestingly, its value $z=1.8288$ (see next section) is significantly different from the optical (Mobasher or BPZ) photometric $z \sim 0.07$. This confirms a significant uncertainty of the photometric redshift estimates, which should therefore be taken with caution. For source $13 \mathrm{~S}$ we have an optical spectrum showing a single line that could be Ly $\alpha$ at redshift 2.94 (see discussion of individual sources).

To better constrain the MAMBO galaxy redshifts photometrically, near-infrared photometry will prove very useful for the typical redshift range (1-3) these objects tend to be found. A forthcoming publication will present such revised estimates along with spectroscopic results for some of the candidate counterparts.

\section{NOTES ON INDIVIDUAL SOURCES}

\subsection{MAMBO Sources with $>4 \sigma$ Significance}

(1) $M M J 100016+021549$.- We tentatively identify a $3.5 \sigma$ radio peak near the MAMBO peak position as the possible counterpart. This radio peak coincides with a very faint optical source near a brighter, irregular source. The radio-mm spectral index $\alpha_{1.4}^{250}=1.00 \pm 0.06$ implies a redshift $\sim 4.1$. The COSMOS photometric redshift catalog gives a redshift of 1.18. Using synthetic templates, HYPERZ yields $z=0.4$ and an early type galaxy. However, the second minimum gives $z=2.8$ and a spiral galaxy template, in better agreement with the radio/mm redshift estimate. This source was also detected with Bolocam at $1.1 \mathrm{~mm}$ (Aguirre et al. 2007), and with SHARC-2 at $350 \mu \mathrm{m}$ at R.A. $10^{\mathrm{h}} 00^{\mathrm{m}} 15.65^{\mathrm{s}}$, decl. $02^{\circ} 15^{\prime} 52^{\prime \prime}$ (Aguirre et al. 2007).

(2) $M M J 100010+021338$.- The mm source appears divided into two components, $2 \mathrm{E}$ and a faint $2 \mathrm{~W}$, with a separation of about one MAMBO beam. The western part is associated with a faint radio source on a bright photometric $z \sim 1$ galaxy. The eastern $\mathrm{mm}$ peak is half a MAMBO beam offset from a radio source that is associated with a galaxy pair of colors suggesting a high redshift, similar to that from a radio/mm estimate. The situation here is confusing: the western $\mathrm{mm}$ peak has a clear optical candidate, the more significant eastern part has no radio sources, and a strong radio sources lies in the middle. Our best guess is that this strong radio source is the $\mathrm{mm}$ source. Because $2 \mathrm{~W}$ is not a significant $\mathrm{mm}$ source in itself, we exclude its radio and optical counterpart from our analysis of the $\mathrm{mm}$ source 
TABLE 5

Colors ANd RedshifTS

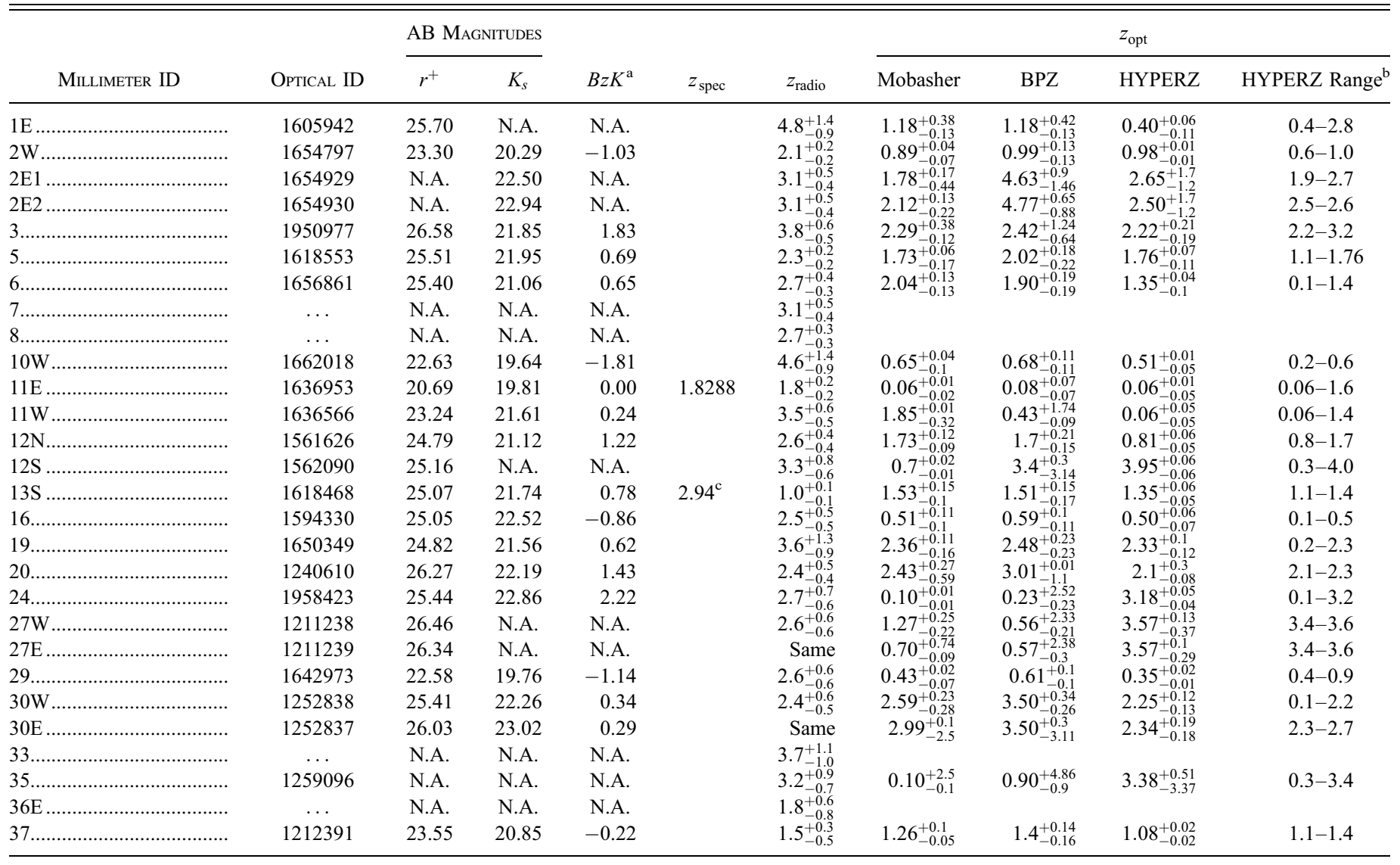

NoтE.-Sources 7, 8, 33, and 36 have optical counterparts in the high-resolution ACS images, but we are currently unable to extract accurate photometry that allows photometric redshift estimates.

${ }^{\mathrm{a}}$ The index $B z K$ is $(z-K)_{\mathrm{AB}}-(B-z)_{\mathrm{AB}}$ (Daddi et al. 2004); see Fig. 9.

${ }^{\mathrm{b}}$ Redshift range of first and second $\chi^{2}$ minima using average and synthetic template SEDs.

${ }^{c}$ Uncertain because based on a single line.

properties summarized in the redshift distribution (Fig. 8), color analysis (Fig. 9), and number counts (Fig. 10).

(3) MM J100057+022013.--There is one clear radio source associated with this MAMBO source, and that is associated with an optical/NIR galaxy. Its radio/mm spectral index of $0.91 \pm$ 0.05 suggests a redshift of 3.3 , which is marginally consistent with the photometric redshift 2.4 of the associated galaxy. This MAMBO source is also detected with Bolocam at $1.1 \mathrm{~mm}$.

(4) $M M J 100007+021149$. - The optical image shows a crowded field of galaxies around the MAMBO source position, but no radio source is found in the field. From the radio $3 \sigma$ upper limit we derive a radio/mm redshift lower limit of $\sim 4$.

(5) $M M J 100031+021241$. - This source has a strong radio counterpart $2^{\prime \prime}$ north of the $\mathrm{mm}$ peak position. The radio source coincides with a faint optical/NIR galaxy with a photometric redshift 1.7-2.0. With a radio/mm spectral index of $0.70 \pm 0.05$ the radio/mm redshift estimate of 2.2 agrees well with the optical redshift estimate.

(6) MM J100008+021304.-The MAMBO source appears fragmented. The main component (6C) has a bright radio source that is $1^{\prime \prime}$ from an optical source pair (blended in the Subaru images but separate in ACS) and coincides with one component of an extended bright $K$ source (Fig. 11). Apparently the radio source counterpart is optically invisible and extremely red. The radio-mm spectral index of $0.77 \pm 0.05 \mathrm{implies}$ a radio $/ \mathrm{mm}$ redshift $\approx 2.5$, consistent with the optical photometric redshift $\sim 2$.
(7) $M M J 100024+021748$. - A substantially elongated (deconvolved $3.1^{\prime \prime} \times 0.5^{\prime \prime}$ ) radio source (COSMOSVLA source 1762, Schinnerer et al. 2007) is found within 2" from the MAMBO peak position. The radio/mm spectral index of $0.83 \pm 0.05 \mathrm{im}-$ plies a redshift of $\approx 2.8$. The radio source centers at the edge of a bright, bluish optical galaxy with photometric redshift $0.32-0.35$. The ACS image shows faint extended emission at the radio peak, which could be a lensed background galaxy.

(8) $M M J 100000+020634$. - This diffuse MAMBO source has a bright radio source at its center. The radio/mm spectral index of $0.77 \pm 0.06$ implies a redshift of $\approx 2.4$. A fragmented optical source is associated with the radio source (Figs. 11 and 12). Photometry is currently not possible due to blending. This source is also detected with Bolocam at $1.1 \mathrm{~mm}$.

(9) MM J100026+021529.- - This mm source shows no radio counterpart. With the $3 \sigma$ upper limit for the radio flux density, we infer $z>3.7$.

(10) MM J095956+021141.-The MAMBO source is diffuse and the radio and optical identification remains ambiguous. Near the central $\mathrm{mm}$ peak there are two possible radio counterparts. The fainter one is located within $1^{\prime \prime}$ from an irregular galaxy with the color of a low-redshift passive galaxy. The radio-mm spectral index $0.98 \pm 0.07$ implies $z=4.0$, whereas the optical photometric redshift $z=0.65-0.68$. The radio source is not centered on the galaxy but coincides with a lump at its western edge. The other, brighter radio source to the southeast does not show an 

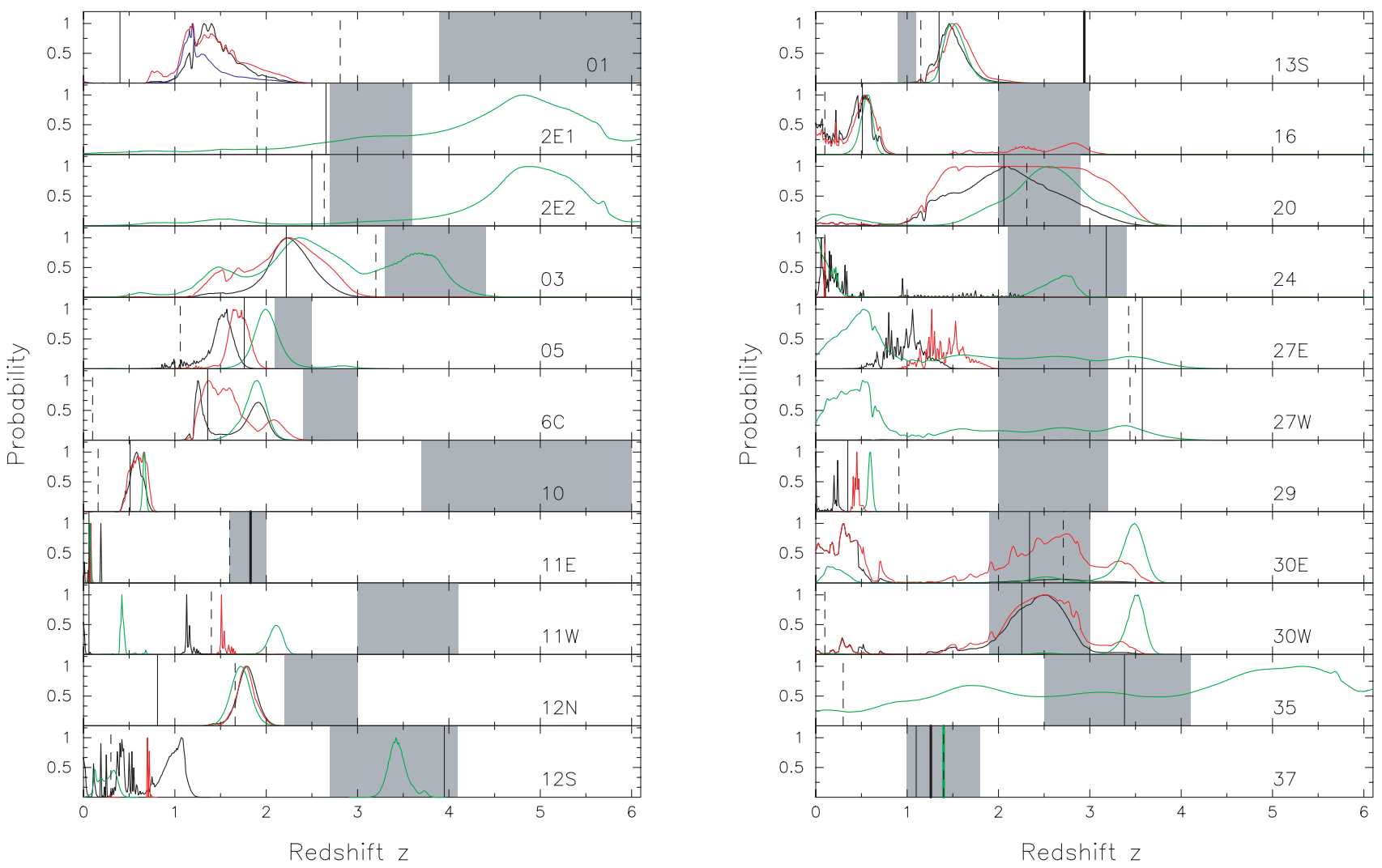

FIG. 7.—Photometric redshift probability distributions for the MAMBO optical counterparts. The curves correspond to different photoredshift codes ( $\$ 2.4$ ): black and red curves are from Mobasher code with and without priors, respectively; green curves from BPZ without priors; a solid and dashed vertical line indicates the HYPERZ first and second minimum, respectively; the shaded areas the radio-mm redshift ranges; and the two thick vertical lines are spectroscopic redshifts. For source 37 we only show the most likely Mobasher and BPZ redshifts through vertical black (1.26) and green (1.4) lines, respectively.

optical or near-IR source. At this point, we do not have a reliable optical counterpart for it.

(11) MM J100038+020825.- This source shows a bright radio counterpart, which is centered on a bright, bluish, compact galaxy with a companion about $1^{\prime \prime}$ to the southwest that apparently also shows radio emission. The radio-mm spectral index of $0.59 \pm 0.04$ implies $z=1.6$ for the brighter component. The optical photometric redshift $\chi^{2}$ distribution shows minima at low $(0.06-0.08)$ and intermediate $(\sim 1.3)$ redshifts. There are three independent spectroscopic redshift measurements, mostly based on a wide (FWHM $\sim 2400 \mathrm{~km} \mathrm{~s}^{-1}$ ) [Mg II] line: $1.8325 \pm$ 0.0023 (Trump et al. 2006), $1.825 \pm 0.002$ (Prescott et al. 2006) and 1.8289 (S. Lilly \& M. Scodeggio 2006, private communication), which when averaged yields $z=1.8288 \pm 0.0037$. This is consistent with the radio photometric redshift and with the second minimum in the optical photoredshift $\chi^{2}$ distribution. The redshift of the fainter companion is unknown. The bright component harbors an X-ray luminous (XID 160) type 1 AGN (Trump et al. 2006); with $L_{\mathrm{X}} \sim 10^{44} \mathrm{erg} \mathrm{s}^{-1}$ (Table 3) it could be classified as a faint QSO.

(12) $M M J 100059+021716$.- This MAMBO source is also detected with Bolocam at $1.1 \mathrm{~mm}$. Three significant radio peaks appear near the MAMBO source position, none of them centered however. The brighter radio source to the northwest of the MAMBO peak shows a starburst-colored optical counterpart with a photometric redshift near that of the radio/mm estimated $z \approx 2.4$. A hardly significant radio source associated with an optical source appears $5^{\prime \prime}$ southwest and a stronger radio source $8^{\prime \prime}$ southeast of the MAMBO peak. We preliminarily identify the northern source as the likely MAMBO counterpart. Right on the MAMBO peak there is a hint of a $\sim 20 \mu \mathrm{Jy}$ radio source associated with an extended faint optical emission.

(13) MM J100015+021244.- This mm source appears elongated north-south with two peaks separated by $12^{\prime \prime}$, but given the low $\mathrm{S} / \mathrm{N}$, this appearance may be due to noise. The southern peak coincides with a strong radio source with a faint multiple optical/K source (Fig. 11).

The radio-mm spectral index of $0.35 \pm 0.09$ implies $z \approx 0.9$, which is consistent with the optical photoredshift. The northern MAMBO peak has no radio counterpart. For the southern radio/ optical source an optical VLT spectrum (S. Lilly 2006, private communication) shows a single line, which could be Ly $\alpha$ at redshift 2.94. If this line was [C $\mathrm{CII}] \lambda 1908$, then $z=1.51$; if [C IV] $\lambda 1546$, then $z=2.10$. The optical and radio photometric redshifts are more consistent with the lower redshifts, $z \sim 1-1.5$.

(14) MMJ100047+021018.-Near the MAMBO position the radio image is confused by a double radio galaxy about $15^{\prime \prime}$ to the south. We identify no clear optical or radio counterpart. From the upper radio flux limit we infer $z>2.6$. This source is detected with Bolocam at $1.1 \mathrm{~mm}$.

(15) MM J100057+021305.-The MAMBO source appears irregular and extended. We identify no radio or optical counterpart. From the upper radio flux limit we infer $z>3.6$.

\subsection{MAMBO Sources with 3-4 $\sigma$ Significance and a Likely Radio Counterpart}

The following sources constitute a radio-selected subsample of the lower significance MAMBO peaks. Because these 


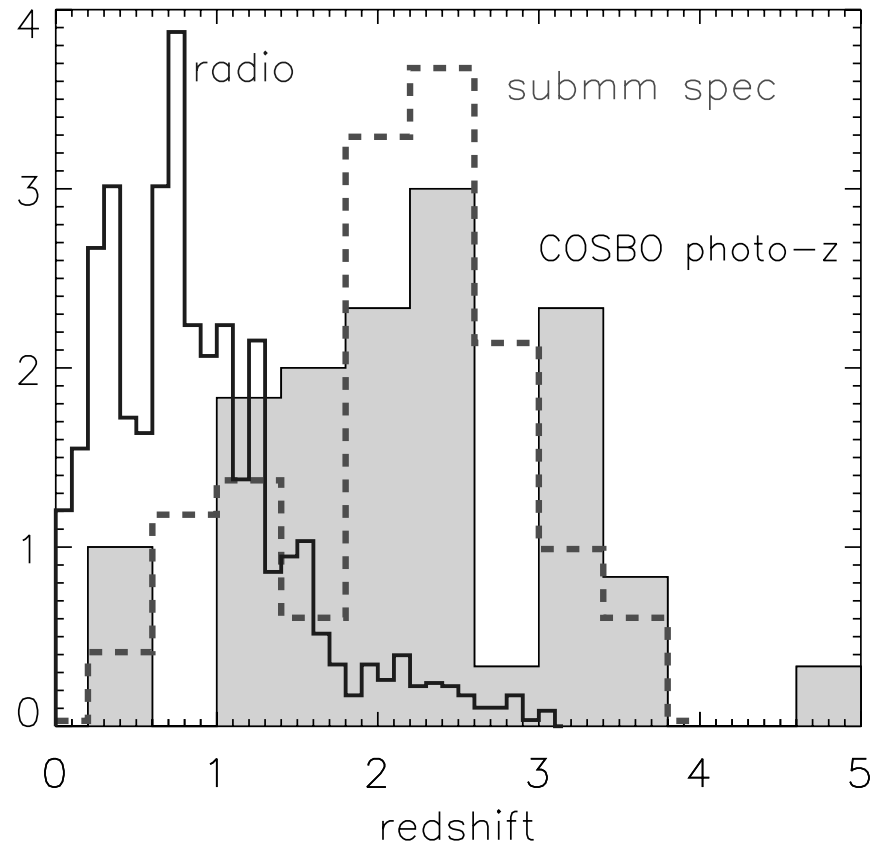

FIG. 8. Optical photometric redshift distribution of the COSMOS sources (gray shaded histogram) compared with the spectroscopic redshifts of radio selected submillimeter sources (dashed line histogram; Chapman et al. 2005), and the photometric redshifts of $<250 \mu \mathrm{Jy}$ radio sources in the COSMOS survey ( finely sampled histogram; Schinnerer et al. 2007). The solid histogram for the COSBO sources includes for each MAMBO source the three photometric redshift estimates (Mobasher, BPZ, HYPERZ), except for 11E, where we use the spectroscopic redshift, and for sources $24,12 \mathrm{~W}$, and 35 , where we ignore the $z<1$ estimates. We show redshifts for only those sources that have secure radio and optical identifications, and photometric data: 2E1, 3, 5, 6, 11E, 13S, 16, 19, $20,24,27 \mathrm{~W}, 30 \mathrm{~W}$, and 37 . The Chapman curve is normalized to the total number of data points in the COSBO sample, the radio sample is scaled to fit on the plot. [See the electronic edition of the Supplement for a color version of this figure.]

MAMBO peaks are associated with a radio source, their reality is more likely than that of a similar significance MAMBO peak without an associated radio source.

(16) $M M J 100056+020841$.- This source shows extended structure in the $\mathrm{mm}$, likely due to noise. We identify a radio source $\sim 4^{\prime \prime}$ from the MAMBO position with an elongated optical counterpart of photometric redshift $\sim 0.5$, with a secondary maximum at $z \sim 2-3$ (Fig. 7). The radio-mm spectral index $0.74 \pm 0.06$ implies $z=2.3$, more consistent with the higher optical redshift maximum. Three arcsec west of the radio source we find an extended, very red object (Fig. 13).

(19) MM J100012+021445.-We identify a faint radio peak near the MAMBO position, coincident with a clumpy optical source.

(20) $M M J 100022+020605$. - A $7 \sigma$ radio source coincides with the MAMBO peak. An optical source is clearly associated with the radio source, which is also identified as a moderately luminous AGN (XID 278) (Table 3). The radio-mm spectral index of $0.72 \pm 0.05$ implies $z \approx 2.8$, which is consistent with the optical photometric redshift.

(24) $M M J 100100+021811$.-A possible radio counterpart is found about $3^{\prime \prime}$ north of the MAMBO peak. The radio source is associated with a red optical/NIR source with a photometric $z \sim 0.1-0.2$, although a second minimum yields a redshift of $\sim 3$.2. The optical source shows extended structure over about $1^{\prime \prime}$, with a bright, unresolved nucleus offset from the optical center and $\sim 0.5^{\prime \prime}$ away from the radio source. The low-redshift solutions appear unlikely. The radio-mm spectral index $0.77 \pm$ 0.07 implies a radio photometric $z \approx 2.4$.

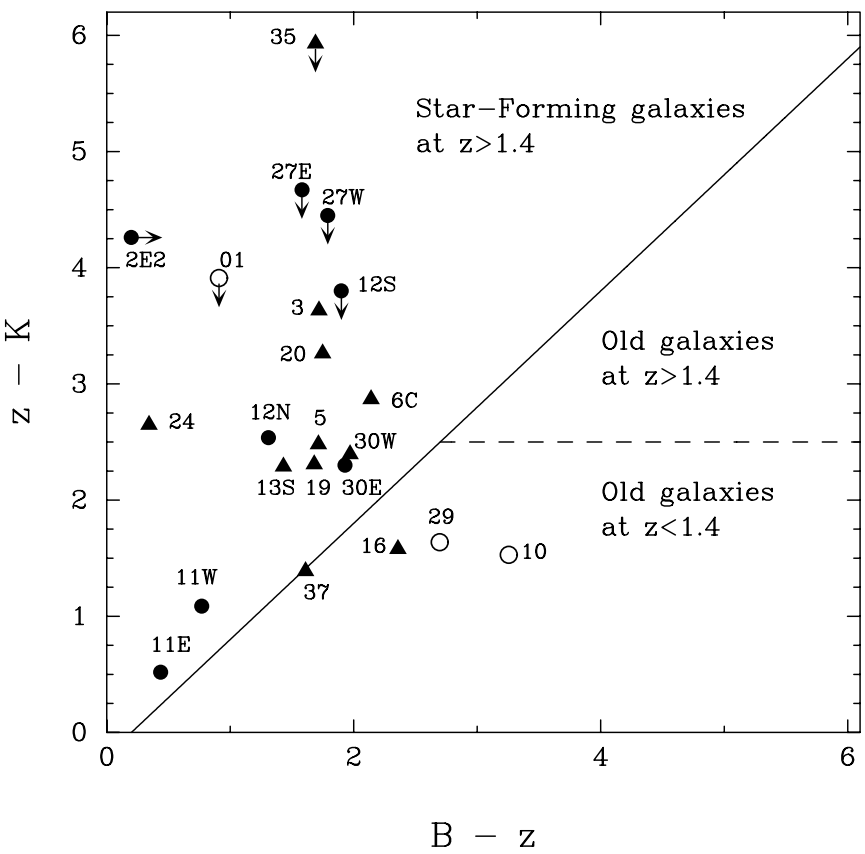

FIG. 9.-Color-color plot using the Subaru $B_{\mathrm{J}}, z^{+}, K_{s}$ photometry of potential MAMBO counterparts identified through a radio source. Filled triangles represent galaxies that have a very likely optical-VLA-MAMBO identification (plus flags in Tables 1 and 2); filled circles represent those with an ambiguous or questionable radio identification ( $o$ in Table 1); and open circles represent those with an unlikely radio identification or ambiguous radio and optical identification. The index $B z K$ in this case is $\left(z^{+}-K_{s}\right)_{\mathrm{AB}}-\left(B_{\mathrm{J}}-z^{+}\right)_{\mathrm{AB}}$ (Daddi et al. 2004), which discriminates between different kind of galaxy populations, independent of reddening: $B z K>-0.2$ (solid line) selects active star-forming galaxies at $z>1.4$, while $B z K<-0.2$ indicates a $z<1.4$ galaxy, except if also $z-K>2.5$ (dashed line), which selects a passive $z>1.4$ galaxy.

(25) MM J095951+021720.-This faint MAMBO source, which is also detected by Bolocam, has a possible faint radio counterpart. The radio-mm spectral index $0.82 \pm 0.06$ would imply $z \approx 2.7$. No optical source is visible at the radio position.

(27) $M M J 100109+020346$. - This diffuse MAMBO source is likely to be associated with a radio source that has a multicomponent optical counterpart seen in the deep Subaru images (Fig. 12), which is identified in the COSMOS PSC as two

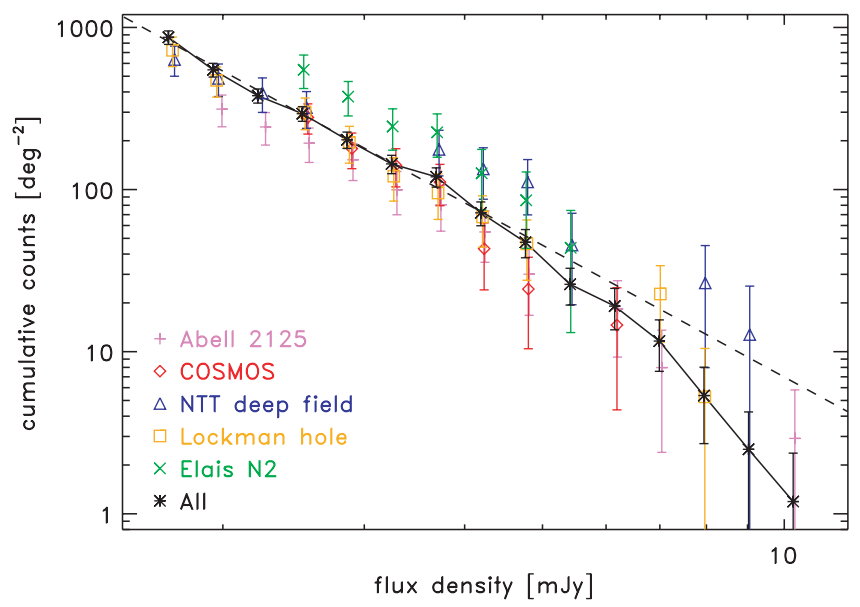

FIG. 10.-Cumulative number counts of $1.2 \mathrm{~mm}$ MAMBO sources from the MAMBO blank field surveys of COSMOS and other fields (Bertoldi et al. 2000; Dannerbauer et al. 2004, 2006; Greve et al. 2004; Voss et al. 2006). The solid line connects the average counts, the dashed line represents a power-law fit (index $-2.7)$ to the lower flux counts. 
01

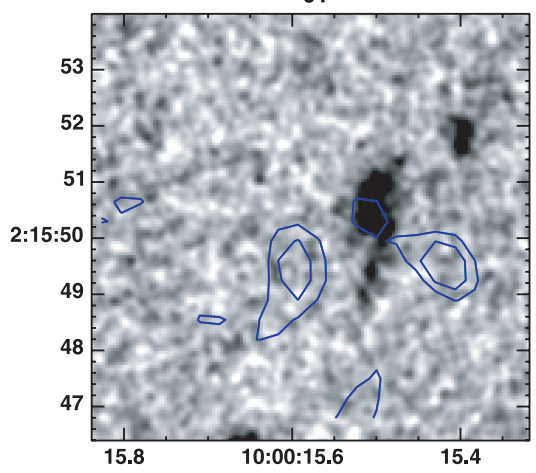

04
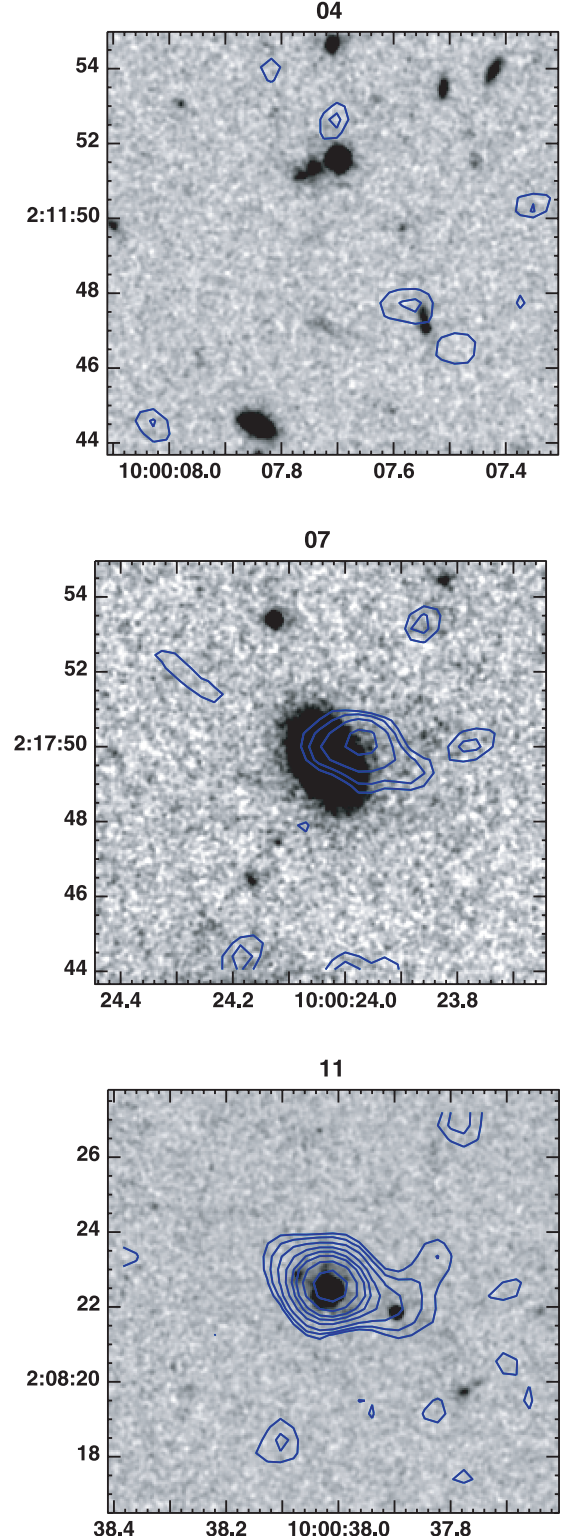
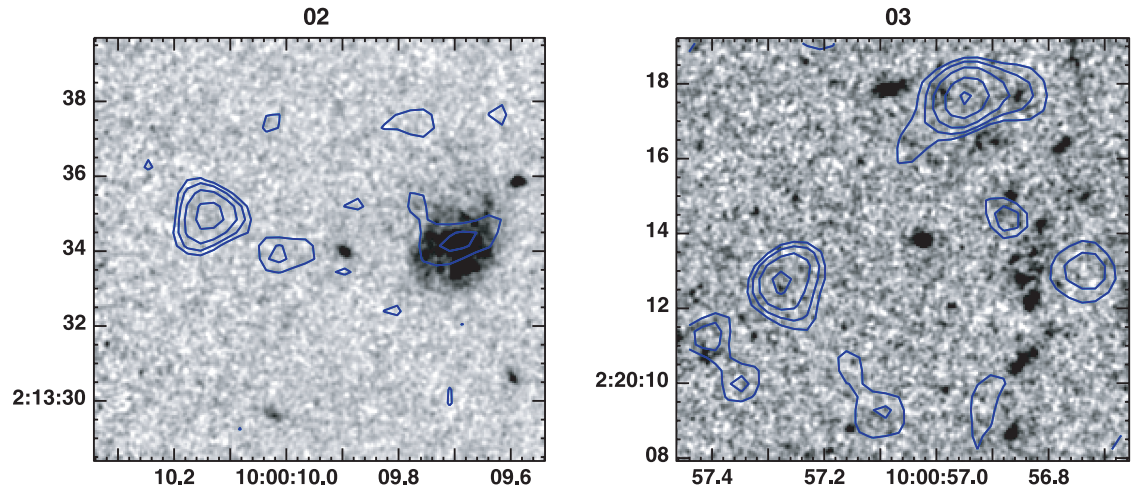

05
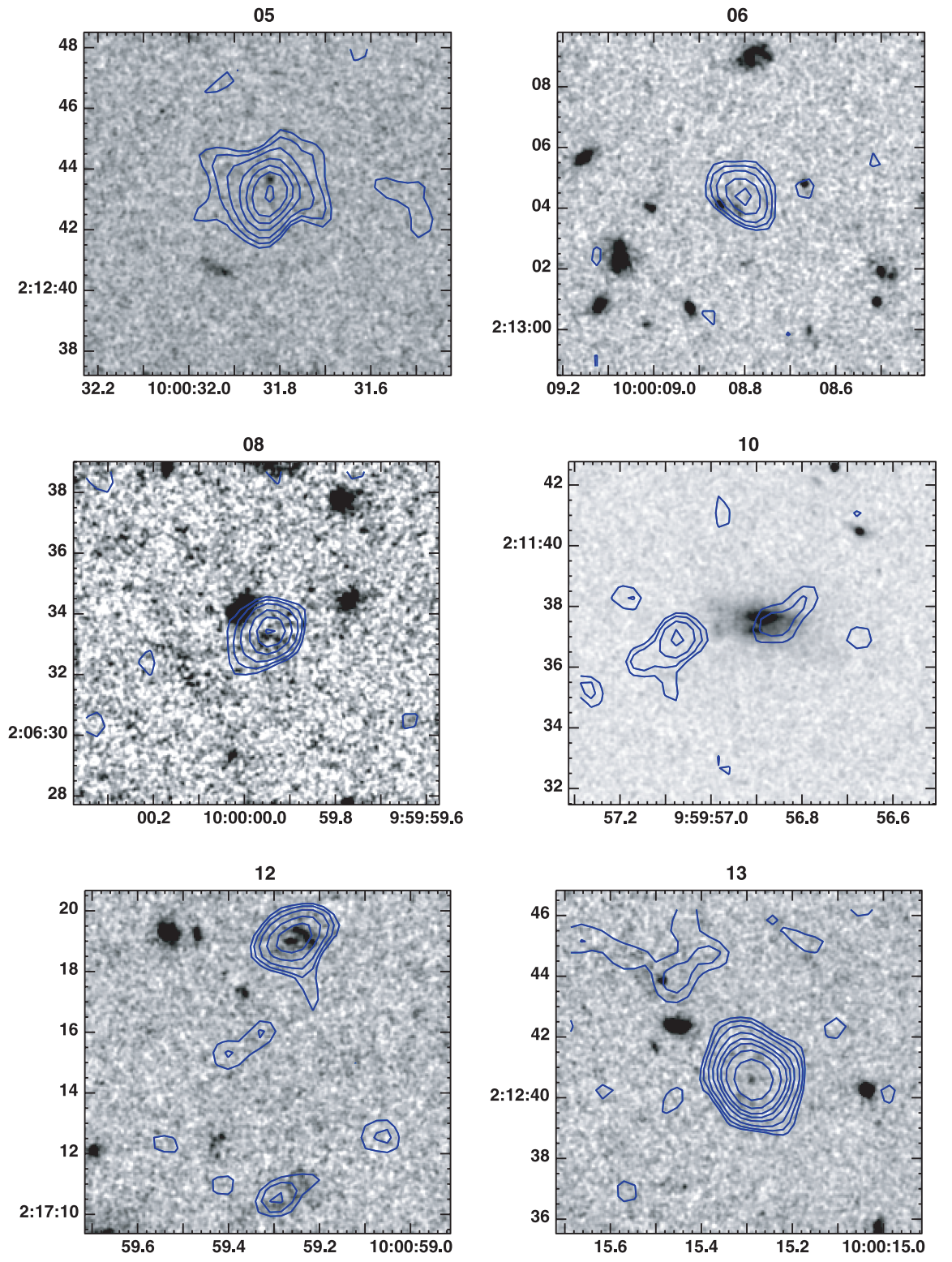

FIG. 11.-HST ACS I-band images of possible radio/optical MAMBO source counterparts. Although the Subaru $i^{+}$data shown in Figs. 6 and 12 are deeper, many of the sources are blended there or do not reveal any structural details. Blue contours show the VLA 1.4 GHz intensities, as in Fig. 6.

sources. The ACS image shows only the eastern part as a compact source. Their optical photometric redshifts $\sim 0.6-1.3$. However, using HYPERZ we obtain redshifts of $\sim 3.5$ for both components, fitting spiral or early-type templates. Based on the faintness and irregular morphology, the high-redshift solution appears more likely. The radio-mm spectral index, $0.75 \pm 0.08$, implies $z \approx 2.3$.

(29) $M M J 100002+021628$. - The radio identification is ambiguous for this faint and somewhat diffuse MAMBO source. The mm source stretches over a dense group of galaxies, many of 
16

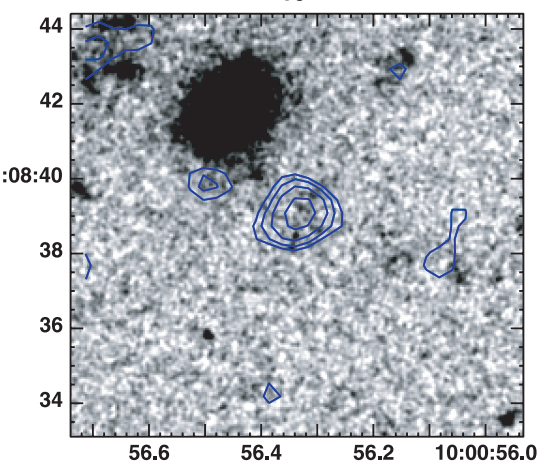

24

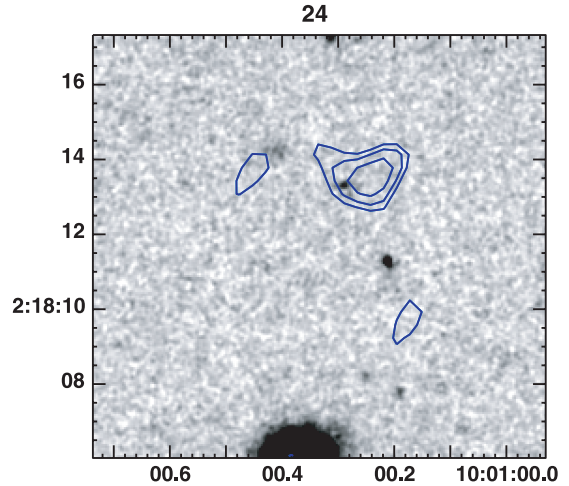

29

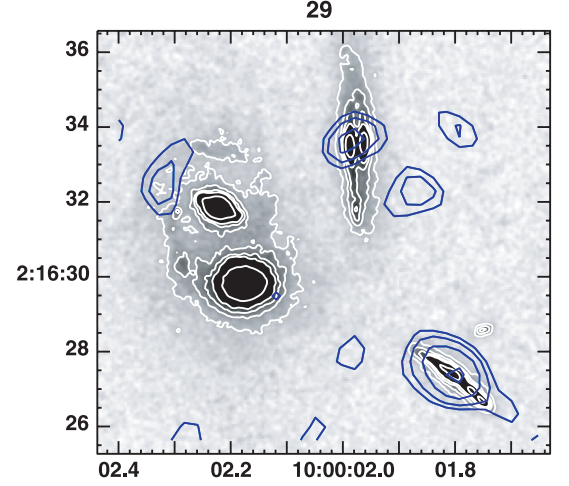

35

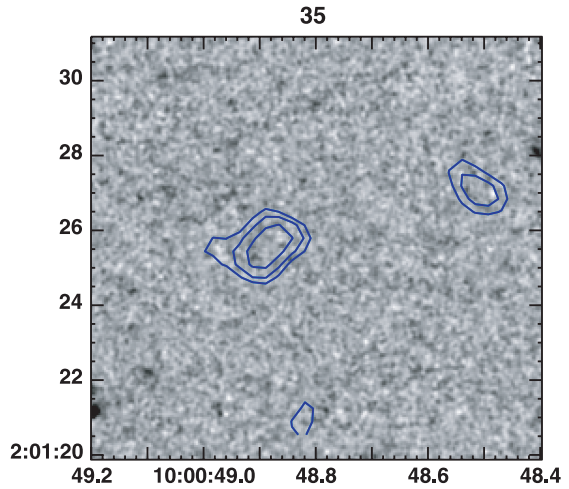

19

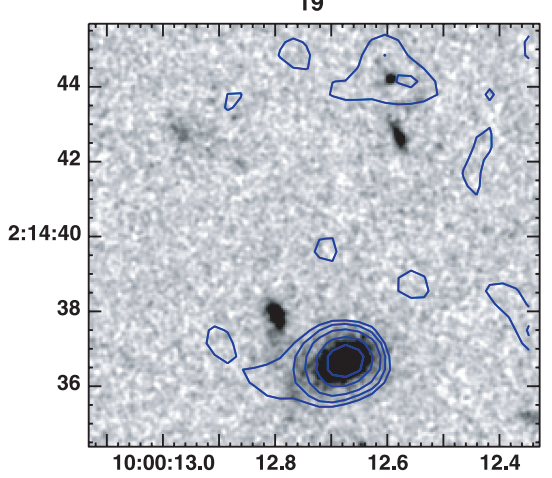

25

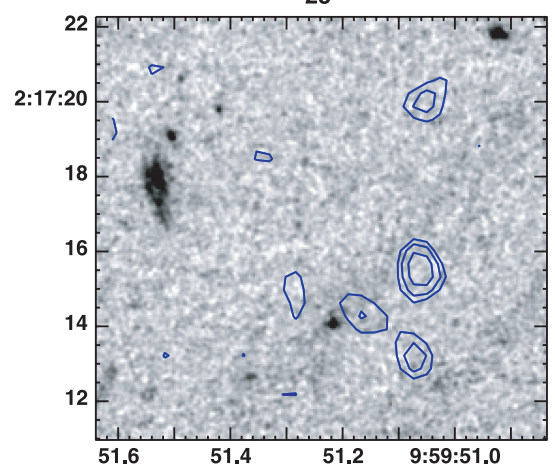

30
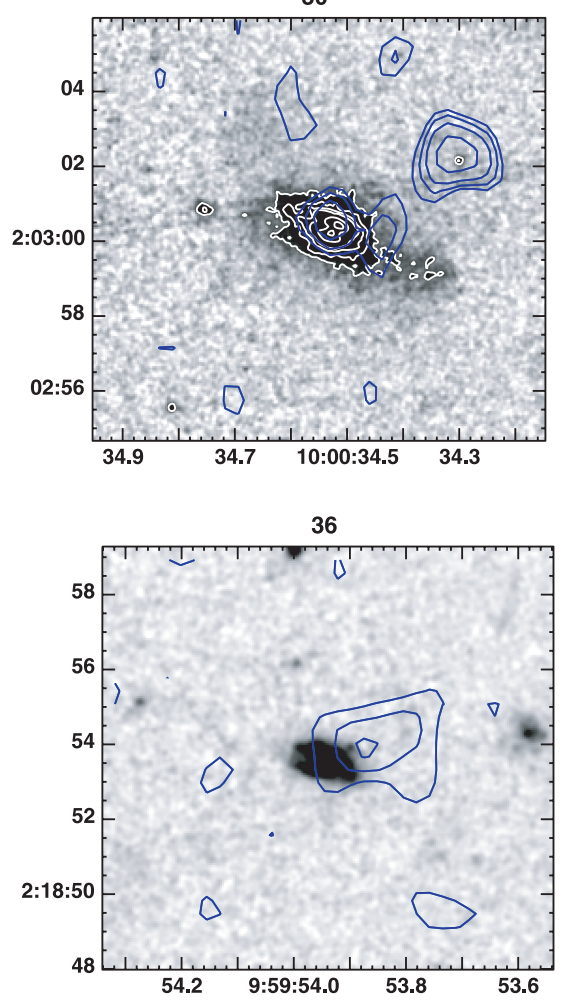

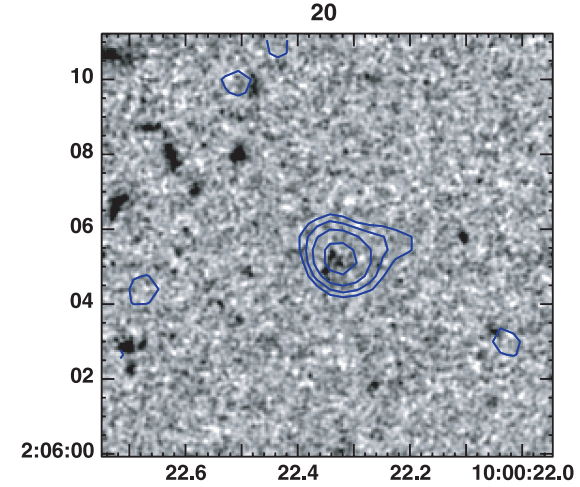

27

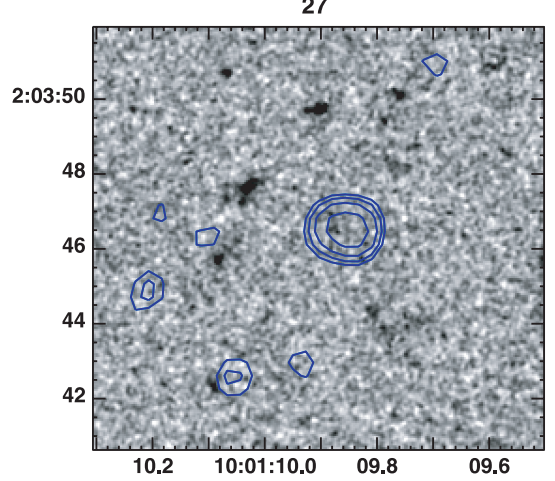

33
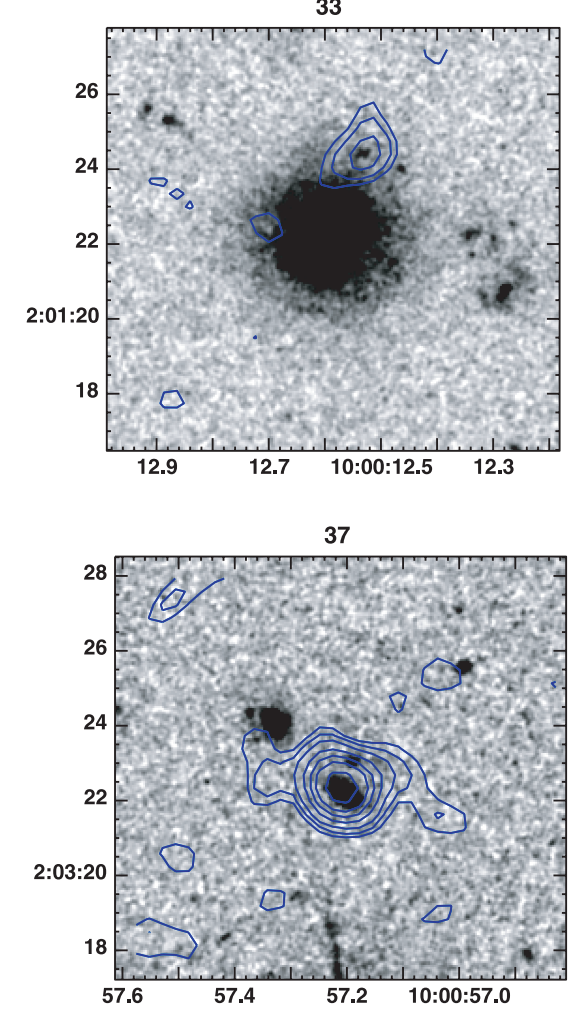

FIG. 11-Continued

which show radio emission. The counterpart to the $\mathrm{mm}$ source remains unclear. The most nearby radio source is located to the southwest and is associated with an elongated, multicomponent (as seen in the ACS image) optical/NIR source of photometric redshift 0.63 . A bright XMM-Newton source (XID 13, Table 3) is associated with a pair of bright optical sources to the northeast, with photometric redshift estimates of 0.5 and 1.5 for its components. There is no radio emission associated with these sources, which is not unusual since the fraction of X-ray sources with radio counterparts at this level is only about $20 \%-25 \%$. 

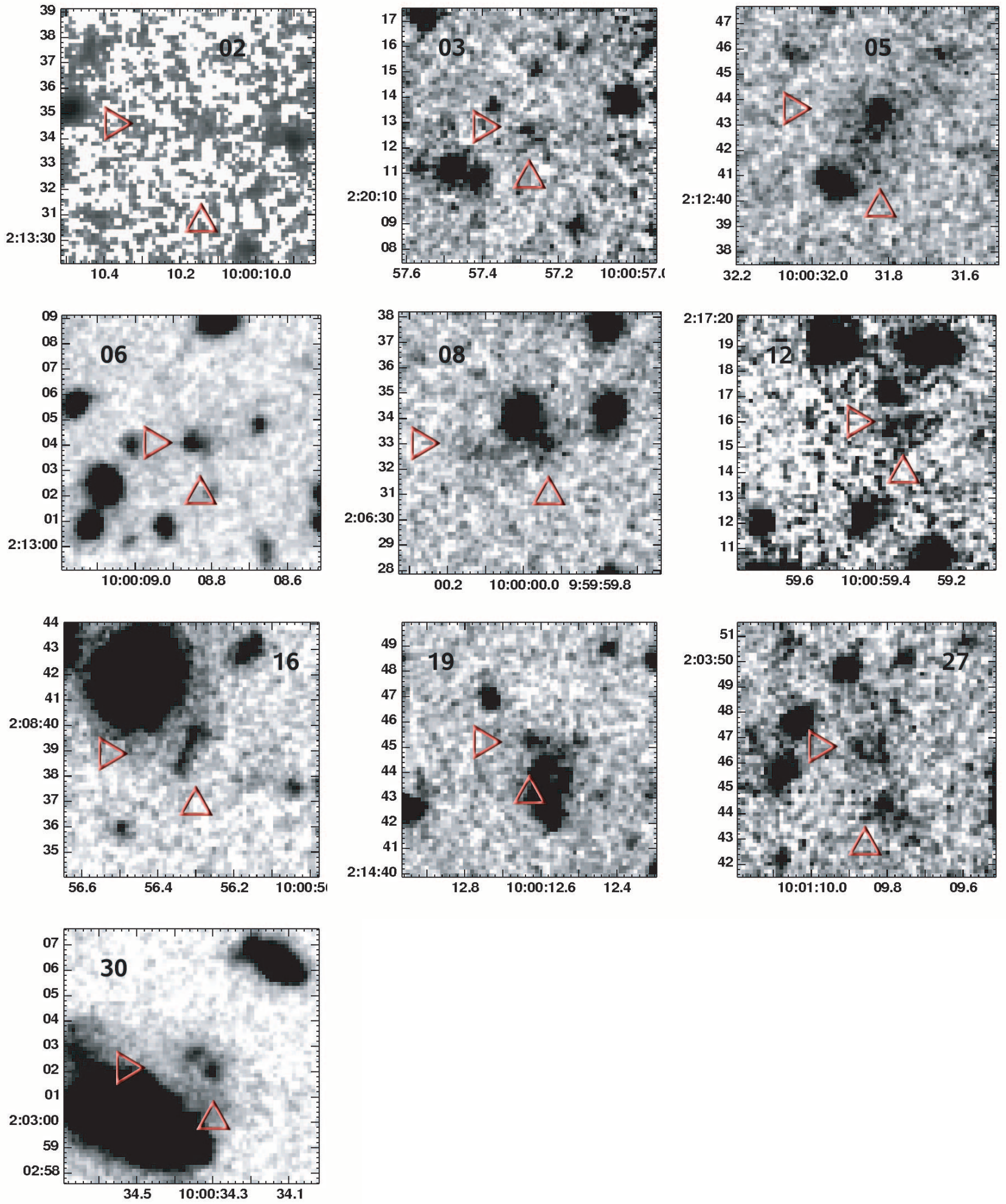

FIG. 12.-Close-up Subaru $i^{+} 10^{\prime \prime} \times 10^{\prime \prime}$ maps centered near possible optical counterparts to radio counterparts, identified through pointers.

(30) $M M J 100034+020304$. - This compact MAMBO source appears related to a radio source that is associated with the southwestern component of a double optical source (Fig. 12) of photometric $z \sim 2.5-3$. The southwest source also appears in the ACS image (Fig. 11). The radio-mm spectral index $0.71 \pm 0.1$ implies $z \approx 2.2$, consistent with the optical redshift.
(33) MM J100012+020125. - This MAMBO source is at the edge of our map and thus, despite its high flux, is of low significance. It is associated with a radio source that has a faint optical counterpart seen in the ACS image, but hardly in the Subaru image because it blends with a large optical galaxy of $z \sim 0.2$. The faint optical source is not in the COSMOS point source catalog 


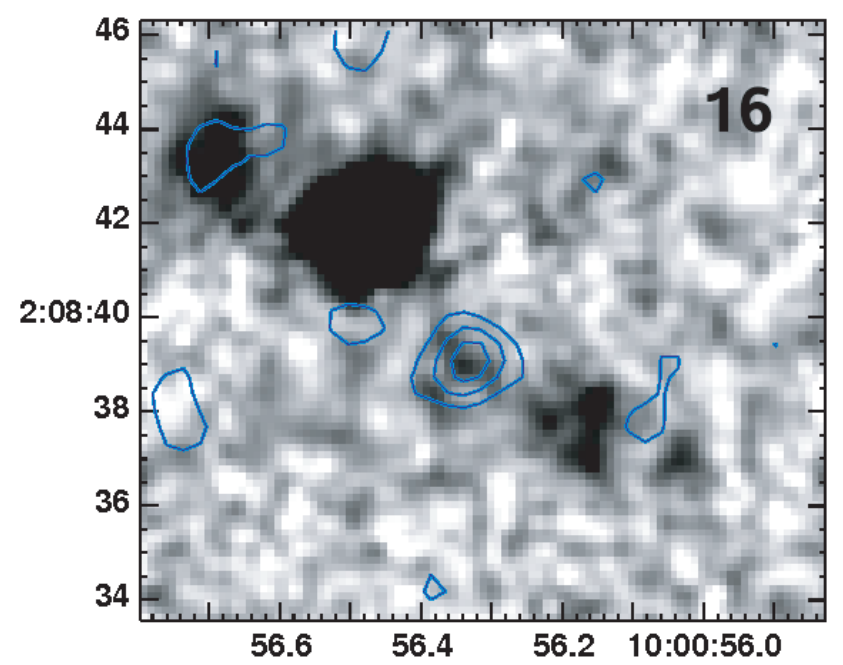

FIG. 13. $-K$-band image of source 16 , overlaid with the VLA radio contours. The radio source, which is the likely MAMBO source counterpart, is neighboring an unusually red source to its southwest.

and we are currently unable to get proper multiband photometry. The radio-mm spectral index $0.9 \pm 0.1$ implies $z \approx 3.3$.

(35) $M M J 100048+020125$. - This $3 \sigma$ MAMBO source is detected with Bolocam. There is an apparent radio and Subaru optical counterpart that is faint and extended on $1^{\prime \prime}$ scale. The Mobasher redshift estimate of 0.1 appears less likely than our HYPERZ solution of 3.4.

\subsection{Faint MAMBO Sources Identified in Bolocam Survey}

We selected the following faint MAMBO sources because they are associated with Bolocam sources. They would have been too insignificant to be selected otherwise.

(36) MM J095953+021851.-The $4 \sigma$ Bolocam source 9 is associated with a east-west double-peaked $3 \sigma$ MAMBO source. We could not identify any radio or optical counterpart in the stronger MAMBO peak, but $\sim 3^{\prime \prime}$ from the fainter peak we find a strong radio source that is associated with a compact object. Due to its proximity to a bright extended galaxy, this compact source is only visible in the ACS image and blended in the Subaru image, thus no photometry is available.

(37) $M M J 100057+020228$. - The $4 \sigma$ Bolocam source 6 is detected near this $2.5 \sigma$ MAMBO double peak, which lies $\sim 6^{\prime \prime}$ from a strong radio source that is the likely counterpart.

\section{DISCUSSION}

\subsection{Number Counts}

Figure 10 shows the cumulative source count at $250 \mathrm{GHz}$ for the COSBO field, as well as for other MAMBO surveys. The counts in the COSBO field are consistent with those seen in previous MAMBO (Greve et al. 2004; Voss et al. 2006) and SCUBA surveys (summarized by Coppin et al. 2006). To compare MAMBO $1.2 \mathrm{~mm}$ counts to SCUBA $850 \mu \mathrm{m}$ counts we look at the results of measurements and models from Eales et al. (2003) and Ivison et al. (2005), who find an wide range of flux ratios $S(850) / S(1200) \approx 2.5 \pm 1.5$ for MAMBO blank field sources that were also observed with SCUBA. High-redshift QSOs show a similar ratio of 2.5, consistent with dust properties similar to local starburst galaxies. Scaling the observed number counts to equivalent $850 \mu \mathrm{m}$ flux densities higher by $\sim 2.5$, the number counts summarized in 13 match those summarized by
Coppin et al. (2006). At $S(1200)<6$ mJy, the MAMBO cumulative counts are well fitted by a power law of index -2.7 , implying a differential source count index of -3.7 . Although the COSMOS data alone do not provide constraints on higher source fluxes, the cumulative counts of all MAMBO blank field surveys show evidence for a steepening of the counts above $6 \mathrm{mJy}$. A steepening is also seen in the SCUBA count at around 6-10 mJy, corresponding to $3-5 \mathrm{mJy}$ at $1.2 \mathrm{~mm}$, i.e., at somewhat lower fluxes than for MAMBO sources. The apparent variance both in the MAMBO and SCUBA counts does not rule out that both results are consistent. The COSMOS counts alone do in fact show a turn at lower flux density $\sim 4 \mathrm{mJy}$. The SCUBA cumulative counts steepen from a power-law slope of -1.4 to -4 , the MAMBO counts from -2.7 to -6.1 , which is, considering the equivalent flux ranges, also consistent.

One may speculate about the nature of the steeping. The steepening $1.2 \mathrm{~mm}$ flux density of $6 \mathrm{mJy}$ relates to sources with FIR luminosities of $\sim 4 \times 10^{13} L_{\odot}$, assuming a standard SED of $\sim 45 \mathrm{~K}$ and $\beta=1.5$. Lehnert \& Heckman (1996) have determined a (self-limiting) maximum FIR surface brightness for starburst galaxies of $\sim 10^{11} L_{\odot} \mathrm{kpc}^{-2}$. In this case, the cutoff in luminosity would imply a maximum size for extreme starburst regions of $\sim 20 \mathrm{kpc}$.

\subsection{Radio Identification}

The radio and FIR luminosities are known to be well correlated for star-forming galaxies (Condon 1992; see also redshift discussion below). Using our deep ( $\sim 7-8 \mu \mathrm{Jy}$ rms $)$ VLA $1.4 \mathrm{GHz}$ data of the central $40^{\prime}$ of the COSMOS field, we have identified 11 of the 15 significant $(\mathrm{S} / \mathrm{N}>4)$ MAMBO sources with radio counterparts above $\sim 25 \mu \mathrm{Jy}$. Our radio detection rate is comparable to those seen in other MAMBO and SCUBA fields with similar radio depth (e.g., Ivison et al. 2005, 2007).

\subsection{Morphologies and Colors}

We find that especially in the deep Subaru images, many of the reliably identified MAMBO galaxies (e.g., 3, 5, 6, 7, 8, 11, 13, 16, and 30) appear with complex morphologies on several arcsec scale (Fig. 12), whereas the high-resolution ACS images (8) tend to show very compact counterparts of the radioidentified MAMBO sources: (e.g., 5, 8, 13, 24, 33, 36).

Such complex morphologies are consistent with, e.g., $H S T$ observations of SCUBA galaxies that often show multicomponent, dusty morphologies, indicative of extended mergers; submillimeter galaxies are typically a factor of $\sim 3$ larger than typical Lyman-break galaxies (Chapman et al. 2004). Highresolution imaging with ALMA will reveal the dust and gas distributions in these systems in exquisite detail. The new highresolution imaging capabilities of the IRAM interferometer might also be able to probe the structure of these objects.

We note that at least three MAMBO sources (source 7, near galaxy at $z \approx 0.32$; source 33 , near galaxy at $z \approx 0.2$; source 36 , near galaxy at $z \approx 0.83$; and possibly source 30 , near galaxy at $z \approx 0.35$ ) show likely counterparts that lie very near large galaxies, and they might therefore be lensed by these foreground objects.

Daddi et al. (2004) showed that independent of their reddening, star-forming and old passively evolving galaxies in the redshift range $1.4<z<2.5$ can be distinguished based on the color index $B z K=(z-K)-(B-Z)$, where $B z K>-0.2$ selects star-forming galaxies. The additional restriction $(z-k)>2.5$ discriminates old passive galaxies at $z>1.4$ from those at lower redshifts. Dannerbauer et al. (2006) showed that (sub-) mm bright 
galaxies may be related to the $B z K$-selected high-redshift starburst population. Figure 9 shows the COSBO galaxy colors in a $z^{+}-K_{s}$ versus $B_{\mathrm{J}}-z^{+}$color-color diagram, which shows that most of them are consistent with the colors of high-redshift starbursts.

Many of the COSBO sources have $K_{s}=20-23$, for which the $\mathrm{Bz} K$ criteria is not well calibrated and must be taken with caution. Eventually, Spitzer photometry will be able to constrain the redshifts at higher redshifts using $R J L$ photometry (Daddi et al. 2004), which will be able to separate star-forming and passive evolving galaxies even with moderate dust extinction.

\subsection{Redshift Distribution}

Figure 8 shows the optical photometric redshift distribution of the 13 most secure radio/optical counterparts. For comparison, we show the spectroscopic redshift distribution for the SCUBA sample of Chapman et al. (2005), and of the faint radio source counterparts in the COSMOS field (Schinnerer et al. 2007). Since averaging the various photometric redshift estimates we have for each source (using the Mobasher, BPZ, and HYPERZ methods) is not necessarily meaningful, we plot for each MAMBO counterpart all three values listed in Table 5 (weighted by $1 / 3$ each), i.e., each source appears three times in Figure 8. This way the uncertainties are adequately reflected in the resulting distribution. For the three cases where significant discrepancies appear between the three optical photometric estimates $(24,27 \mathrm{~W}$, and 35), we judge that from their optical faintness, irregular morphology, and the large radio-mm redshift, the low-redshift solutions are unlikely, and we therefore ignore the $z<1$ estimates in the distribution, but adequately weight the others into the distribution. For 11E we use the spectroscopic redshift instead of the photometric estimates.

We find that the redshift distribution for the COSMOS sample (including lower limits for radio nondetections) is similar to that found for previous SCUBA or MAMBO surveys, with a peak at $z \sim 2.5$, and most of the sources being between $z \sim 1.5$ and 3 (e.g., Dannerbauer et al. 2004; Pope et al. 2005). The distribution of optical photometric redshifts basically agrees with the radio-photometric distribution, with a slight excess at $z \sim 0.5-1$.

This low-redshift excess could arise from a false identification of the corresponding radio counterpart, or an incorrect photometric redshift of the optical counterpart to the radio identification. In fact, there are often multiple minima in the $\chi^{2}$ photoredshift distribution, with a minimum both at low redshift and close to the higher radio-FIR photoredshift. We also find that the minima are quite sensitive to the choice of templates, and we therefore determined photometric redshifts using three different sets of templates.

Overall, we conclude that determining the redshifts for these sources remains a complex issue, but that the current sample is consistent with previous estimates for submillimeter galaxies, with most of the sources between $z=2$ and 3. Again, this issue should become clearer using the Spitzer IRAC data (Sanders et al. 2007) and with ongoing spectroscopy of the most likely counterparts.

\section{5. $A G N s$}

Two of the MAMBO sources were detected in X-rays at the depth of the COSMOS XMM-Newton observations (Brusa et al. 2007; Table 3). The implied X-ray luminosities for these two sources are $\geq 10^{43}-10^{44} \mathrm{erg} \mathrm{s}^{-1}$, indicating moderately luminous AGNs. Given the still quite shallow X-ray coverage of the field, we probe only sources with $L_{\mathrm{X}}>10^{43} \mathrm{erg} \mathrm{s}^{-1}$ at $z \sim 2$ (see Fig. 14 of Trump et al. 2006) and therefore a significant fraction of MAMBO sources can still harbor a moderately luminous AGN below our detection limit. Thus, these results imply X-ray upper luminosity limits for the rest of the MAMBO sources of a few $\times 10^{43} \mathrm{erg} \mathrm{s}^{-1}$. For comparison, the FIR luminosity (corresponding to thermal emission from warm dust) for the typical MAMBO source in our sample is $>10^{45} \mathrm{erg} \mathrm{s}^{-1}$. Overall, the data for the majority of the sample are consistent with the conclusions by Alexander et al. (2005) that for the large majority of (sub)mm galaxies the dominant energy source for the far-IR emission is star formation.

\subsection{Blank Sources}

We have identified four sources $(4,9,14$ [Bolocam 29], 15) with robust MAMBO detections $(>4 \sigma)$ that have no radio counterpart above a flux density of $24 \mu \mathrm{Jy}$. These sources may be the most interesting in the sample, representing the most distant $(z>3.5)$ extreme starburst galaxies. Such distant systems set the most stringent limits on galaxy formation models (Greve et al. 2005). Again, the Spitzer data may prove very valuable to identify these sources. It is interesting to keep in mind that the large inverse- $K$-correction for the submillimeter galaxies implies that we could as easily detect a source out to cosmic reionization $(z \geq 6)$ as we could detect a source at $z \sim 1$.

The research of the Bonn-Heidelberg group was supported through a grant from the Deutsche Forschungsgemeischaft within the focus program SPP1177. K. J. acknowledges support by the German DFG under grant SCHI 536/3-1. We gratefully acknowledge the contributions of the entire COSMOS collaboration consisting of more than 70 scientists. More information on the COSMOS survey is available at http://www.astro.caltech .edu/ cosmos. We thank Mara Salvato and the anonymous referee for helpful comments.

Facilities: IRAM (30 m), HST (ACS), VLA, Subaru, XMM, CSO (Bolocam), KPNO (FLAMINGOS)

\section{REFERENCES}

Alexander, D., Bauer, F. E., Chapman, S. C., Smail, I., Blain, A. W., Brandt, W. N., \& Ivison, R. J. 2005, ApJ, 632, 736

Aguirre, J. E., et al. 2007, ApJS, submitted

Benítez, N. 2000, ApJ, 536, 571

Bertoldi, F., et al. 2000, A\&A, 360, 92

Blain, A., Smail, I., Ivison, R. J., Kneib, J.-P., \& Frayer, D. T. 2002, Phys. Rep., 369,111

Bolzonella, M., Miralles, J.-M., \& Pelló, R. 2000, A\&A, 332, 135

Brusa, M., et al. 2007, ApJS, 172, 353

Bruzual, G., \& Charlot, S. 2003, MNRAS, 344, 1000

Calzetti, D., Armus, L., Bohlin, R. C., Kinney, A. L., Koornneef, J., StorchiBergmann, T. 2000, ApJ, 533, 682

Capak, P., et al. 2007, ApJS, 172, 99

Carilli, C. L., \& Yun, M. S. 1999, ApJ, 513, L13

. 2000, ApJ, 530, 618

Chapman, S., et al. 2003, ApJ, 585, 57

Chapman, S. C., Blain, A., Smail, I., \& Ivison, R. 2005, ApJ, 622, 772

Chapman, S. C., Smail, I., Windhorst, R., Muxlow, T., \& Ivison, R. 2004, ApJ,

611,732

Coleman, G. D., Wu, C.-C., \& Weedman, D. W. 1980, ApJS, 43, 393 
Condon, J. J. 1992, ARA\&A, 30, 575

Coppin, K., et al. 2006, MNRAS, 372, 1621

Daddi, E., Cimatti, A., Renzini, A., Fontana, A., Mignoli, M., Pozzetti, L., Tozzi, P., \& Zamorani, G. 2004, ApJ, 617, 746

Dannerbauer, H., Lehnert, M. D., Lutz, D., Tacconi, L., Bertoldi, F., Carilli, C., Genzel, R., \& Menten, K. M. 2004, ApJ, 606, 664

Dannerbauer, H., et al. 2006, ApJ, 637, L5

Downes, A. J. B., Peacock, K. A., Savage, A., \& Carrie, D. R. 2004, MNRAS, 218,31

Dunlop, J. 2001, NewA Rev., 45, 609

Eales, S., Bertoldi, F., Ivison, R., Carilli, C., Dunne, L., \& Owen, F. 2003, MNRAS, 344, 169

Genzel, R., Baker, A. J., Tacconi, L. J., Lutz, D., Cox, P., Guilloteau, S., \& Omont, A. 2003, ApJ, 584, 633

Greve, T. R., Ivison, R. J., Bertoldi, F., Stevens, J. A., Dunlop, J. S., Lutz, D., \& Carilli, C. L. 2004, MNRAS, 354, 779

Greve, T., et al. 2005, MNRAS, 359, 1165

Hasinger, G., et al. 2007, ApJ, 172, 29

Ivison, R. J., et al. 2002, MNRAS, 337, 1

2005, MNRAS, 364, 1025

2007, MNRAS, in press (astro-ph/0702544)
Kreysa, E., et al. 1998, Proc. SPIE, 3357, 319

Lehnert, M. D., \& Heckman, T. M. 1996, ApJ, 472, 546

Mobasher, B., et al. 2007, ApJS, 172, 117

Neri, R., et al. 2003, ApJ, 597, L113

Peacock, J., et al. 2001, Nature, 410, 169

Pope, A., Borys, C., Scott, D., Conselice, C., Dickinson, M., \& Mobasher, B. 2005, MNRAS, 358, 149

Pope, A., et al. 2006, MNRAS, 370, 1185

Prescott, M. K. M., Impey, C. D., Cool, R. J., \& Scoville, N. Z. 2006, ApJ, 644, 100

Sanders, D., et al. 2007, ApJS, 172, 86

Schinnerer, E., et al. 2004, AJ, 128, 1974 2007, ApJS, 172, 46

Schuecker, P., et al. 2001, A\&A, 368, 86

Scoville, N., et al. 2007, ApJS, 172, 38

Scott, S. E., et al. 2002, MNRAS, 331, 817

Tacconi, L. J., et al. 2006, ApJ, 640, 228

Taniguchi, Y., et al. 2007, ApJS, 172, 9

Trump, J. R., et al. 2006, ApJS, 165, 1

Voss, H., Bertoldi, F., Carilli, C., Owen, F. N., Lutz, D., Holdaway, M., Ledlow, M., \& Menten, K. M. 2006, A\&A, 448, 823 